\title{
Quantum Filter Diagonalization with Compressed Double-Factorized Hamiltonians
}

\author{
Jeffrey Cohn, ${ }^{1, *}$ Mario Motta, ${ }^{1, \dagger}$ and Robert M. Parrish ${ }^{2, \dagger}$ \\ ${ }^{1}$ IBM Quantum, IBM Research-Almaden, San Jose, California 95120, USA \\ ${ }^{2}$ QC Ware Corporation, Palo Alto, California 94301, USA
}

(Received 7 May 2021; revised 8 September 2021; accepted 3 November 2021; published 15 December 2021)

\begin{abstract}
We demonstrate a method that merges the quantum filter diagonalization (QFD) approach for hybrid quantum-classical solution of the time-independent electronic Schrödinger equation with a low-rank double factorization (DF) approach for the representation of the electronic Hamiltonian. In particular, we explore the use of a novel sparse "compressed" double factorization (C-DF) truncation of the Hamiltonian within the time-propagation elements of QFD, while retaining a similarly compressed but numerically converged double-factorized representation of the Hamiltonian for the operator expectation values needed in the QFD quantum matrix elements. The new C-DF method is found to provide substantial additional compression at any given accuracy metric over the traditional "explicit" double factorization approach. Together with significant circuit reduction optimizations and number-preserving postselection and echosequencing error mitigation strategies, the method is found to provide accurate predictions for low-lying eigenspectra in a number of representative molecular systems, while requiring reasonably short circuit depths and modest measurement costs. The method is demonstrated by experiments on noise-free simulators, simulations including models of decoherence and shot-noise, and real quantum hardware.
\end{abstract}

DOI: 10.1103/PRXQuantum.2.040352

\section{INTRODUCTION}

Solving the many-particle Schrödinger equation to compute eigenpairs of a Hamiltonian operator is an important application in computational science. For example, it arises in the simulation of the electronic structure of molecules and materials, as well as in mathematical optimization problems. In the context of classical computation, different strategies are employed to numerically determine approximate ground and excited Hamiltonian eigenpairs, typically by assuming that eigenstates have a certain structure.

Digital quantum computers have been proposed as an alternative and complementary approach to the determination of approximate ground and excited Hamiltonian eigenstates. While efficient ground- and excited-state determination cannot be guaranteed for a generic Hamiltonian, since this is a QMA-complete problem [1], a wealth of heuristic quantum algorithms have been designed and

\footnotetext{
*jeffrey.cohn@ibm.com

†mario.motta@ibm.com

‡rob.parrish@qcware.com
}

Published by the American Physical Society under the terms of the Creative Commons Attribution 4.0 International license. Further distribution of this work must maintain attribution to the author(s) and the published article's title, journal citation, and DOI. demonstrated in recent years. Simulation of the timedependent Schrödinger equation, on the other hand, is a more natural application for a quantum computer, as it lies in the bounded-error quantum polynomial time (BQP) complexity class [2]. This observation has generated an increasingly intense research effort, aimed at integrating the simulation of the time-dependent Schrödinger equation in the structure of quantum computational algorithms for eigenstate determination [3-5].

Quantum filter diagonalization (QFD) (and two similar methods developed simultaneously in the literature) $[3,4,6]$ is a quantum algorithm in which a Hamiltonian operator is projected on a subspace spanned by a set of nonorthogonal quantum states generated via approximate quantum time evolution (or other quantum circuit propagation), and postfacto classically diagonalized. QFD can be regarded to as a quantum computational equivalent of classical filter diagonalization $[7,8]$, from which it inherits the connection with the Lanczos algorithm. Furthermore, it is an example of a quantum subspace diagonalization method [6,9-11] in which, starting from a set of approximate reference states for the targeted eigenvectors that can be easily prepared classically, a basis for a subspace is constructed using time propagation.

The implementation of QFD on contemporary quantum hardware poses a number of conceptual and technical challenges. Among them, (i) the high gate complexity 


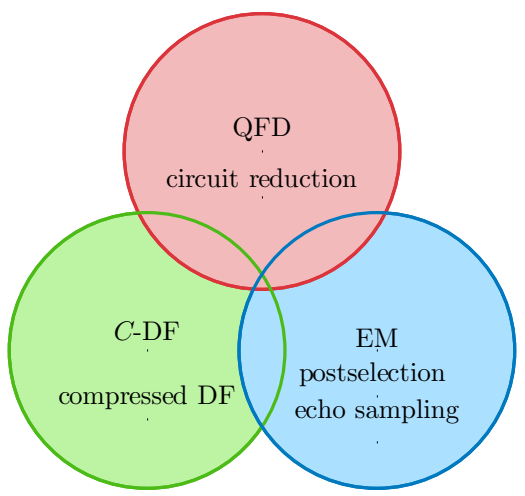

FIG. 1. Schematic representation of the techniques explored in the present work: quantum filter diagonalization (QFD) is combined with a compressed double factorization (C-DF) approximation of the electronic Hamiltonian, and a set of error mitigation (EM) techniques.

required by the simulation of time evolution under the Hamiltonian, which is especially pronounced for the electronic structure problem, (ii) the need of evaluating off-diagonal matrix elements that define overlap and Hamiltonian matrices, which requires Hadamard or swap quantum circuits, and (iii) the integration of dedicated error mitigation techniques in the structure of the algorithm.

In the present work, we demonstrate a technique that merges QFD with a compressed low-rank double factorization of the electronic structure Hamiltonian, to achieve substantially shortened circuit representations of the time propagation steps, and to economize the evaluation of offdiagonal matrix elements. Furthermore, we introduce a combination of postselection and echo-sequencing aimed at mitigating errors from violations of particle number and spin- $z$ conservation. The proposed techniques, sketched in Fig. 1, are demonstrated using classical simulators of quantum devices, and performing experiments on IBM quantum hardware.

\section{METHODS}

\section{A. "Compressed" double-factorized electronic Hamiltonian}

A key technical element of this manuscript is the "compressed" double factorization (C-DF) approximate representation of the electronic Hamiltonian, which provides for reduced gate count requirements in quantum circuits for QFD time propagation and reduced measurement requirements for Hamiltonian expectation values. Below, we review the representation of the electronic Hamiltonian in the double-factorized representation as previously discussed by several authors in the literature [12-19], including the popular "explicit" double factorization procedure $[14,16,17,19]$ (X-DF) for numerically finding the tensor factors. We then develop a new C-DF procedure for numerically finding the tensor factors with enhanced compression and accuracy.

\section{The electronic Hamiltonian}

In a real, orthonormal, and spin-restricted orbital basis [spatial parts of each orbital $\left\{\phi_{p}\left(\vec{r}_{1}\right)\right\}$ the same for each spin orbital $\left\{\psi_{p, \alpha}\left(\vec{x}_{1}\right) \equiv \phi_{p}\left(\vec{r}_{1}\right) \alpha\left(s_{1}\right)\right\}$ and $\left\{\psi_{p, \beta}\left(\vec{x}_{1}\right) \equiv\right.$ $\left.\left.\phi_{p}\left(\vec{r}_{1}\right) \beta\left(s_{1}\right)\right\}\right]$, the electronic Hamiltonian may be written as [20]

$$
\hat{H} \equiv E_{\mathrm{Ext}}+\sum_{p q}(p|\hat{\kappa}| q) \hat{E}_{p q}^{+}+\frac{1}{2} \sum_{p q r s}(p q \mid r s) \hat{E}_{p q}^{+} \hat{E}_{r s}^{+},
$$

where the singlet spin-summed one-particle substitution operator is $\hat{E}_{p q}^{+} \equiv \sum_{p q, \sigma} \hat{p}_{\sigma}^{\dagger} \hat{q}_{\sigma}$. Here $\hat{p}_{\sigma}^{\dagger}\left(\hat{p}_{\sigma}\right)$ is a fermionic creation (annihilation) operator for spatial orbital $p$ and spin label $\sigma$.

The spatial orbital electron repulsion integral (ERI) tensor is written in chemists' notation as

$$
(p q \mid r s) \equiv \iint_{\mathbb{R}^{6}} d \vec{r}_{1} d \vec{r}_{2} \phi_{p}\left(\vec{r}_{1}\right) \phi_{q}\left(\vec{r}_{1}\right) \frac{1}{r_{12}} \phi_{r}\left(\vec{r}_{2}\right) \phi_{s}\left(\vec{r}_{2}\right) .
$$

The modified spatial-orbital one-particle integrals are

$$
(p|\hat{\kappa}| q) \equiv(p|\hat{h}| q)-\frac{1}{2} \sum_{r}(p r \mid q r) .
$$

The spatial-orbital one-particle Hamiltonian integrals are

$$
\begin{aligned}
(p|\hat{h}| q) \equiv & \left(p\left|-\nabla_{1}^{2}\right| q\right)+\left(p\left|-\sum_{A} Z_{A} / r_{1 A}\right| q\right) \\
& +2 \sum_{i}(p q \mid i i)-\sum_{i}(p i \mid q i),
\end{aligned}
$$

where $\left\{\vec{r}_{A}\right\}$ are the nuclear positions, $\left\{Z_{A}\right\}$ are the corresponding nuclear charges, and $\left\{\phi_{i}\left(\vec{r}_{1}\right)\right\}$ are the core spatial orbitals in an active space picture. The external system self-energy is

$$
E_{\mathrm{Ext}} \equiv \sum_{A>B} \frac{Z_{A} Z_{B}}{r_{A B}}
$$

The external system self-energy, one-particle Hamiltonian integrals, and electron repulsion integrals are polynomially tractable input quantities from classical electronic structure codes.

When naïvely expanded into Pauli words in, e.g., the Jordan-Wigner representation, this operator appears to require $\mathcal{O}\left(n_{p}^{4}\right)$ unique Pauli words (here $n_{p}$ is the number of spatial orbitals), with significant classical efforts required to lower the prefactor and/or scaling by merging multiple commuting Pauli words into a minimal set of noncommuting measurement groups $[21,22]$. It has recently been 
empirically demonstrated that this grouping can reduce the scaling of the number of noncommuting measurement groups to $\mathcal{O}\left(n_{p}^{3}\right)$ [21] Even this reduced scaling implies significant computational cost in time propagation or evaluation of the expectation value of this Hamiltonian. Recently, a number of authors have achieved substantial practical reductions in this cost of both of these considerations through an approach we will generically refer to as "double factorization" (DF) [12,14,16-19,23]. The crux of the idea is a representation of the ERI tensor as

$$
(p q \mid r s) \approx \sum_{t} \sum_{k} \sum_{l} U_{p k}^{t} U_{q k}^{t} Z_{k l}^{t} U_{r l}^{t} U_{s l}^{t},
$$

where the "leaf tensor" $U_{p k}^{t}$ is constrained to be orthonormal (actually, we require this matrix to be special orthogonal without loss of generality for all cases encountered in this work),

$$
\sum_{k} U_{p k}^{t} U_{q k}^{t}=\delta_{p q}, \quad \sum_{k} U_{k p}^{t} U_{k q}^{t}=\delta_{p q}, \quad \text { for all } t,
$$

and the "core tensor" $Z_{p q}^{t}$ is constrained to be symmetric,

$$
Z_{k l}^{t}=Z_{l k}^{t} \quad \text { for all } t
$$

In Eq. (5), the sum over $t$ goes up to a maximum value of $n_{\mathrm{DF}}$. The value of $n_{\mathrm{DF}}$ is a user-specified parameter of the DF fitting procedure, and can either be specified by the user or determined automatically to achieve a user-specified error tolerance in the approximate ERI tensor, e.g., similar to what is done in density fitting and Cholesky decomposition, respectively. We elect to use the former approach and explicitly specify $n_{\mathrm{DF}}$ for all examples herein. Similarly, we can take the orthonormal eigendecomposition of

$$
(p|\hat{\kappa}| q)=\sum_{k} U_{p k}^{0} f_{k}^{0} U_{q k}^{0} .
$$

Here only a single expansion index is needed due to the analytical nature of the eigendecomposition (in contrast to $n_{\mathrm{DF}}$ expansion indices for the ERI tensor above) and is denoted by the special index "0." Provided that efficient methods exist [24-26] to transform the representation of the one-particle orbital basis according to the orbital transformation $U_{p k}^{t}$, the Hamiltonian may now be written as

$$
\hat{H} \equiv E_{\mathrm{Ext}}+\sum_{k} f_{k}^{0} \hat{E}_{k k}^{+}(0)+\frac{1}{2} \sum_{t} \sum_{k l} Z_{k l}^{t} \hat{E}_{k k}^{+}(t) \hat{E}_{l l}^{+}(t) .
$$

The operators $\hat{E}_{k k}^{+}(t)$ are diagonal in qubit representations such as the Jordan-Wigner and parity representations. Therefore, the expectations over separate $k$ and $l$ terms can be measured simultaneously for each $t$, drastically reducing the number of required measurements to evaluate Hamiltonian expectation values. Similarly, the implementation of time propagation (Trotterized across $t$ ) can be accomplished by commuting and highly parallelized controlled- $\hat{Z}$ rotations. The generic idea of double factorization can therefore substantially reduce the cost of quantum circuit implementation of quantum chemistry methods. However, the specific practical cost of implementation will depend on the number of $t$ factors required to achieve an accurate factorization of the ERI tensor, i.e., on $n_{\mathrm{DF}}$. We try to reduce this cost in the methodology developed below.

\section{2. "Explicit" double factorization}

In the straightforward "explicit" approach to double factorization (which we label as X-DF) [14,16,17,19], we first eigendecompose the ERI tensor into a form of density fitting factorization:

$$
(p q \mid r s)=\sum_{t} V_{p q}^{t} \lambda_{t} V_{r s}^{t}
$$

with $V_{r s}^{t}=V_{s r}^{t}$ (up to numerical noise in accidental degeneracies). Next, for each eigenvector, we eigendecompose

$$
V_{r s}^{t}=\sum_{k} U_{p k}^{t} U_{q k}^{t} \gamma_{k}^{t}
$$

and then form

$$
Z_{k l}^{t} \equiv \gamma_{k}^{t} \lambda_{t} \gamma_{l}^{t}
$$

Note that the only approximation in X-DF involves the number of terms $n_{\mathrm{DF}}$ retained in the sum over $t$ in Eq. (10) - the subsequent eigendecomposition of Eq. (11) is lossless. In principle, all terms can be retained, leading to zero error at the cost of $\mathcal{O}\left(n_{p}^{2}\right)$ scaling of $n_{\mathrm{DF}}$. However, it is well known from resolution of the identity or synonymously density fitting (RI-used to avoid the unfortunate elision with double factorization's DF), Cholesky decomposition (CD), and general eigenstructure studies that the eigenvalues of the ERI tensor decay roughly geometrically with rank, allowing for practical approximate truncation with $\mathcal{O}\left(n_{p}\right)$ scaling of $n_{\mathrm{DF}}$ for any reasonable user-specified error tolerance. Unfortunately, even this advantageous level of truncation retains $\mathcal{O}\left(n_{p}^{3}\right)$ scaling information in the $U_{p k}^{t}$ tensors (as with RI or CD), with a numerically redundant form of $Z_{k l}^{t}$ as seen in Eq. (12). One could imagine that it might be possible to push below the $\mathcal{O}\left(n_{p}\right)$ scaling limit in $n_{\mathrm{DF}}$ (or at the very least, lower the prefactor at this scaling) by allowing both $U_{p k}^{t}$ and $Z_{k l}^{t}$ to be unconstrained in rank structure, as has been done previously in nonorthogonal tensor hypercontraction (THC). We numerically attempt to move toward this more-compressed factorization in the next section. 


\section{3. "Compressed" double factorization}

The X-DF procedure provides a straightforward and explicit recipe for obtaining the double-factorized quantities $U_{p k}^{t}$ and $Z_{k l}^{t}$, but it may be a nonoptimal factorization due to the nested form of the eigendecompositions. Here, we consider an alternative C-DF based on global optimization of a least-squares objective function.

The least-squares objective function for C-DF is

$$
\begin{aligned}
& \mathcal{O}\left(U_{p k}^{t}, Z_{k l}^{t}\right) \\
& \quad \equiv \frac{1}{2}\left|(p q \mid r s)-\sum_{t} \sum_{k l} U_{p k}^{t} U_{q k}^{t} Z_{k l}^{t} U_{r l}^{t} U_{s l}^{t}\right|_{\mathcal{F}}^{2},
\end{aligned}
$$

where $\mathcal{F}$ denotes the vector-type Frobenius norm. Defining

$$
\Delta_{p q r s} \equiv(p q \mid r s)-\sum_{t} \sum_{k l} U_{p k}^{t} U_{q k}^{t} Z_{k l}^{t} U_{r l}^{t} U_{s l}^{t}
$$

then the gradients are

$$
\frac{\partial \mathcal{O}}{\partial Z_{k l}^{t}}=-\sum_{p q r s} \Delta_{p q r s} U_{p k}^{t} U_{q k}^{t} U_{r l}^{t} U_{s l}^{t}
$$

and

$$
\frac{\partial \mathcal{O}}{\partial U_{p k}^{t}}=-4 \sum_{q r s l} \Delta_{p q r s} U_{q k}^{t} Z_{k l}^{t} U_{r l}^{t} U_{s l}^{t} .
$$

Note that the recent paper on the Jastrow-Factor variational quantum eigensolver (VQE) [18] briefly considers in an appendix direct fitting of the doubly factorized tensors of the ERI tensor to the exact ERI tensor, which goes beyond X-DF in the direction of C-DF. However, no details are given as to the numerical procedure used to perform this fit.

Unconstrained form in terms of orbital rotation generators.- -To remove the orthogonality constraints, one can always define the special orthogonal orbital rotation matrices $U_{p k}^{t}$ in terms of matrix exponentials of antisymmetric orbital rotation generator matrices $X_{p k}^{t}$ :

$$
U_{p k}^{t} \equiv\left[\exp \left(\hat{X}^{t}\right)\right]_{p k}
$$

Here

$$
\hat{X}^{t} \equiv X_{p q}^{t}|p\rangle\langle q|
$$

subject to

$$
X_{p q}^{t}=-X_{q p}^{t} \quad \text { for all } t
$$

In Eq. (17), the notation $\exp (\hat{M})$ means the matrix exponential of the matrix operator symbolically defined as
$\hat{M}$. In this form, the C-DF objective function becomes unconstrained,

$$
\mathcal{O}\left(U_{p k}^{t}, Z_{k l}^{t}\right) \rightarrow \mathcal{O}\left(X_{p q}^{t}, Z_{k l}^{t}\right),
$$

and the gradient of the C-DF objective function is easily evaluated through the chain rule, yielding

$$
\frac{\partial \mathcal{O}}{\partial X_{k^{\prime} l^{\prime}}^{t}}=\sum_{p k} \frac{\partial \mathcal{O}}{\partial U_{p k}^{t}} \frac{\partial U_{p k}^{t}}{\partial X_{k^{\prime} l^{\prime}}^{t}}
$$

Efficient linear algebraic operations for the matrix exponential and the matrix exponential gradient exist in the form of the Wilcox identity [27]. These have been specialized to the cases of antisymmetric generators $\hat{X}$, and may be considered to be universal library functions for any $X_{p k}^{t}$. With this unconstrained formulation, one may supply the objective function and analytical gradient function to a numerical unconstrained continuous optimizer such as limited-memory Broyden-Fletcher-Goldfarb-Shanno algorithm (L-BFGS), and numerically optimize the $X_{p k}^{t}$ and $Z_{k l}^{t}$ factors of C-DF simultaneously. We have implemented this and found that while it provides a straightforward and simple approach, convergence can be markedly slow. For this reason, we pursue a nested "two-step" C-DF fitting procedure below.

Core tensor analytical fitting.-An interesting avenue to explore is the form of the fitting equations when the factors $U_{p k}^{t}$ are known (in analogy to the least-squares tensor hypercontraction procedure [28] in nonorthogonal tensor hypercontraction, in which an analytical formula for $Z_{k l}$ resulted):

$$
\mathcal{O}\left(Z_{k l}^{t} \mid U_{p k}^{t}\right)=\frac{1}{2}\left|(p q \mid r s)-\sum_{t} \sum_{k l} U_{p k}^{t} U_{q k}^{t} Z_{k l}^{t} U_{r l}^{t} U_{s l}^{t}\right|_{\mathcal{F}}^{2} .
$$

Here the weak form of the objective function is

$$
\frac{\partial \mathcal{O}}{\partial Z_{k l}^{t}}=-\sum_{p q r s} \Delta_{p q r s} U_{p k}^{t} U_{q k}^{t} U_{r l}^{t} U_{s l}^{t}=0 \quad \text { for all } k, l, t .
$$

Expanding yields

$$
\sum_{t^{\prime}} \sum_{k^{\prime} l^{\prime}} M_{k k^{\prime}}^{t t^{\prime}} Z_{k^{\prime} l^{\prime}}^{t^{\prime}} M_{l l^{\prime}}^{t t^{\prime}}=R_{k l}^{t} \quad \text { for all } k, l, t,
$$

where

$$
R_{k l}^{t} \equiv \sum_{p q r s} U_{p k}^{t} U_{q k}^{t}(p q \mid r s) U_{r l}^{t} U_{s l}^{t}
$$


and

$$
\begin{aligned}
M_{k k^{\prime}}^{t \prime^{\prime}} & \equiv\left[\sum_{p} U_{p k}^{t} U_{p k^{\prime}}^{t^{\prime}}\right]\left[\sum_{q} U_{q k}^{t} U_{q k^{\prime}}^{t^{\prime}}\right] \\
& =\left[\sum_{p} U_{p k}^{t} U_{p k^{\prime}}^{t^{\prime}}\right]^{2} \\
& \equiv\left[S_{k k^{\prime}}^{t t^{\prime}}\right]^{2} .
\end{aligned}
$$

The "2" notation is the elementwise square in the last expression. The $\hat{S}_{k k^{\prime}}^{t t^{\prime}}$ are metric matrices (symmetric, positive definite, with singular values in $[0,1]$ ) when unrolled in $k t \times k^{\prime} t^{\prime}$. The $M_{k k^{\prime}}^{t t^{\prime}}$ are thus also metric matrices, with the extra specialization of having wholly positive values.

These equations can be effectively written as

$$
\sum_{t^{\prime} k^{\prime} l^{\prime}} A_{t k l, t^{\prime} k^{\prime} l^{\prime}} Z_{t^{\prime} k^{\prime} l^{\prime}}=R_{t k l} \quad \text { for all } k, l, t
$$

where

$$
A_{t k l, t^{\prime} k^{\prime} l^{\prime}} \equiv M_{k k^{\prime}}^{t t^{\prime}} M_{l l^{\prime}}^{t t^{\prime}},
$$

i.e., a simple set of linear equations. It should be noted that the matrix $\hat{A}$ has formal singularities of degeneracy $n_{\mathrm{DF}}-$ 1 , and may also contain numerical near singularities if the active $U_{p k}^{t}$ matrices are numerically similar. In practice, the eigendecomposition-based Moore-Penrose pseudoinverse approach to solve these equations costs $\mathcal{O}\left[\left(n_{\mathrm{DF}} n_{p}^{2}\right)^{3}\right]$, which is tractable for medium-sized problems. Moreover, conjugate gradient and L-BFGS approaches appear to also provide reliable convergence with lowered cost. The matrix-vector product primitive needed for such iterative approaches is

$$
\sigma_{t k l} \equiv \sum_{t^{\prime} k^{\prime} l^{\prime}} A_{t k l, t^{\prime} k^{\prime} l^{\prime}} b_{t^{\prime} k^{\prime} l^{\prime}}
$$

This can be efficiently implemented in terms of matrix multiplications with a cost of $\mathcal{O}\left(n_{\mathrm{DF}}^{2} n_{p}^{3}\right)$.

Two-step C-DF fitting.- The above finding of an analytical fit for $Z_{k l}^{t}$ for any proposed $U_{p k}^{t}$ leads to the following "two-step" C-DF fitting:

$$
\mathcal{O}\left(U_{p k}^{t}\right)=\frac{1}{2}\left|(p q \mid r s)-\sum_{t} \sum_{k l} U_{p k}^{t} U_{q k}^{t} Z_{k l}^{\downarrow t} U_{r l}^{t} U_{s l}^{t}\right|_{\mathcal{F}}^{2} .
$$

Here $Z_{k l}^{\downarrow t}$ is shorthand for the optimal $Z_{k l}^{t}$ predicated on the current $U_{p k}^{t}$ discussed in the section above. In practice, one actually works with the unconstrained form $\mathcal{O}\left(X_{p q}^{t}\right)$ within this two-step C-DF fitting procedure, with the unconstrained optimization in $X_{p q}^{t}$ being handled by L-BFGS. The explicit two-step C-DF procedure is as follows.
Stage 0 . Use the X-DF factorization to obtain a guess for the factors $\left\{U_{p k}^{t}\right\}$ and $\left\{Z_{k l}^{t}\right\}$.

Stage 1 . For $\left\{U_{p k}^{t}\right\}$ from stage 0 , find the globally optimal $\left\{Z_{k l}^{t}\right\}$ via least squares (analytical). Note that the stage- $1 U_{p k}^{t}$ tensor is the same as in stage 0 , but the stage- $1 Z_{k l}^{t}$ tensor is different, and in particular does not have the highly constrained rank structure of Eq. (12), leading to a positive definite improvement in the approximation at stage 1 .

Stage 2. For $U_{p k}^{t}$ from stage 0 or stage 1 (identical), run two-step C-DF fitting to find the globally optimal $\left\{U_{p k}^{t}\right\}$ and $\left\{Z_{k l}^{t}\right\}$.

Note that the $U_{p k}^{t}$ "leaf tensor" is the same in stage 0 and stage 1 , but the $Z_{k l}^{t}$ "core tensor" changes between stage 0 (where it is definitionally numerically redundant) and stage 1 (where it is permitted to be full rank). Both tensors change from stage 1 to stage 2, though the $U_{p k}^{t}$ and $Z_{k l}^{t}$ tensors from stage 1 can be used as a guess to seed the optimization process of stage 2 . This procedure is analogous to the "two-step complete active space self-consistent field" method, where the orbitals are rotated and optimized in an outer loop, with the active space configuration interaction exactly solved at each orbital point in an inner loop $[20,29]$.

\section{Example numerical performance of C-DF: integral metrics}

The C-DF approach outlined above was implemented in a simple PYTHON numpy environment. L-BFGS (scipy) with analytical gradients is used to drive the optimization loop in $X_{p k}^{t}$. The matrix exponential needed to form $U_{p k}^{t}$ is evaluated from the complex Hermitian eigendecomposition of $i X_{p k}^{t}$, and the corresponding exponential derivative is evaluated by the Wilcox formula [27]. The linear equations used to solve for the optimal $Z_{p k}^{t}$ for a given $U_{p k}^{t}$, e.g., Eq. (27), are solved explicitly via an eigendecomposition-based Moore-Penrose pseudoinverse with eigenvalue cutoff of $10^{-10}$.

Figure 2 shows representative performance of the X-DF and C-DF methods. The test case is a 10 orbital active space of the lowest lying $\pi$ and $\pi^{*}$ orbitals of naphthalene, with the orbitals computed at restricted Hartree-Fock at cc-pVDZ level of theory (RHF/cc-pVDZ). The C-DF objective function $\mathcal{O}\left(U_{p k}^{t}, Z_{k l}^{t}\right)$ and the maximum absolute deviation (MAD) in the ERI tensor $\left|\Delta_{p q r s}\right|_{\infty}$ are plotted as a function of DF rank expansion $n_{\mathrm{DF}}$ for the stage- 0 , stage1 , and stage- 2 outcomes of the C-DF procedure. Stage 0 is the result that would be obtained by the older X-DF approach, while stage 2 corresponds to a complete C-DF procedure. 


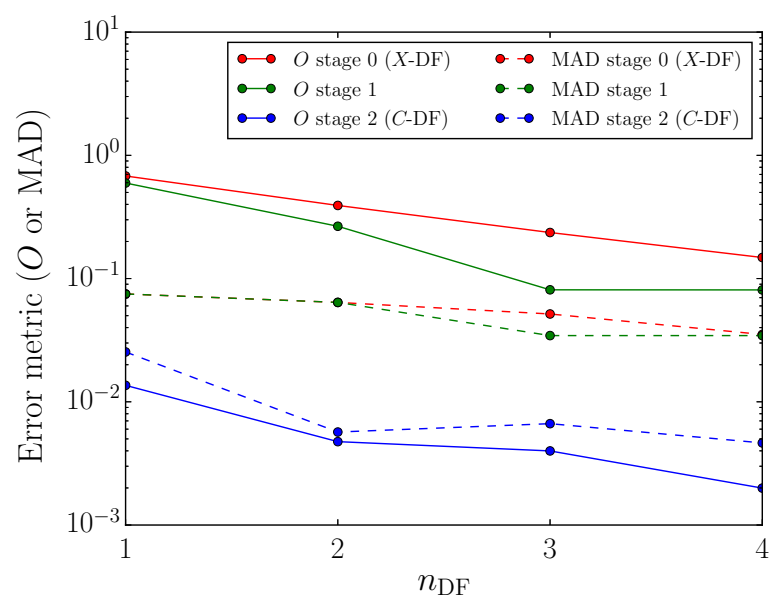

FIG. 2. Error metrics for stage-0 (X-DF), stage-1, and stage-2 (C-DF) two-step double factorization approaches. The test case is naphthalene with an $n_{p}=10$ active space of low-lying $\pi$ and $\pi^{*}$ orbitals computed at RHF/cc-pVDZ. Here $O$ is the C-DF least-squares objective function, and MAD is the mean absolute deviation $\left|\Delta_{p q r s}\right|_{\infty}$.

The results are generally straightforward. Using the shortest possible DF factorization $n_{\mathrm{DF}}=1$, the stage- $0 \mathrm{X}$ DF obtains a rather coarse representation of the ERI tensor, with objective function value $\delta$ of $\mathcal{O}\left(10^{0}\right)$ and ERI MAD of $\mathcal{O}\left(10^{-1}\right)$. Adding more X-DF factors monotonically improves matters, with roughly geometric convergence (as expected from density fitting); however, the prefactor is rather slow, and only a fourfold reduction in objective function value and ERI MAD are achieved by $n_{\mathrm{DF}}=4$. Moving to stage 1 , one finds that refitting the $Z_{k l}^{t}$ factors with fixed X-DF $U_{k l}^{t}$ factors does not significantly improve matters. However, moving to the full stage-2 CDF method, one finds substantial improvement of at least one order of magnitude in $O$ and roughly one order of magnitude in $\delta$ for all $n_{\mathrm{DF}}$. Particularly striking is the fact that it is better to use the coarsest $n_{\mathrm{DF}}=1 \mathrm{C}$-DF factorization than to use the largest $n_{\mathrm{DF}}=4$ factorization shown here.

\section{Example numerical performance of C-DF: energy errors and scaling trends}

At the request of a reviewer, we have elected to perform an extended study of the error characteristics of $\mathrm{X}$-DF versus $\mathrm{C}$-DF in the context of chemically meaningful observables such as total adiabatic state energies computed at full configuration interaction (FCI) within the DF-truncated Hamiltonian. For the first part of the study, we have elected to use the $\pi / \pi^{*}$ active spaces of alltrans linear alkenes taken from restricted Hartree-Fock at STO-3G level of theory (RHF/STO-3G) orbitals - similar results are obtained with linear acenes and several other classes of test systems, but the linear alkenes provide more datapoints that are tractable with FCI [here we study linear alkenes with up to $K=6$ double bonds (dodecahexene) involving active spaces up to $(12 \mathrm{e}, 12 \mathrm{o})$ ], thus providing a better view of the trends.

Figure 3 shows the error characteristics as a function of $n_{\mathrm{DF}}$ for the $(12 \mathrm{e}, 12 \mathrm{o})$ minimal $\pi / \pi^{*} \mathrm{RHF} / \mathrm{STO}-3 \mathrm{G}$ active space of $(K=6)$-double-bond dodecahexene. Here, we find strong concordance between error characteristics in the ERI tensor (top panel) and error characteristics in the total FCI energies for the lowest singlet or triplet state (lower panel). For this test case, X-DF exhibits slow but regular convergence with seemingly geometric character in both the ERI and total energy characteristics. Note that state energy errors are still $>100 \mathrm{mE}_{h}$ even for $n_{\mathrm{DF}}=6$. By contrast C-DF errors are always at least $10 \times$ smaller than X-DF errors, and the improvement increases with $n_{\mathrm{DF}}$, i.e., C-DF converges faster than X-DF for this test case. Note however that the C-DF convergence is not regular, and may even be slightly nonmonotonic due to the nonvariational characteristics of C-DF and/or numerical
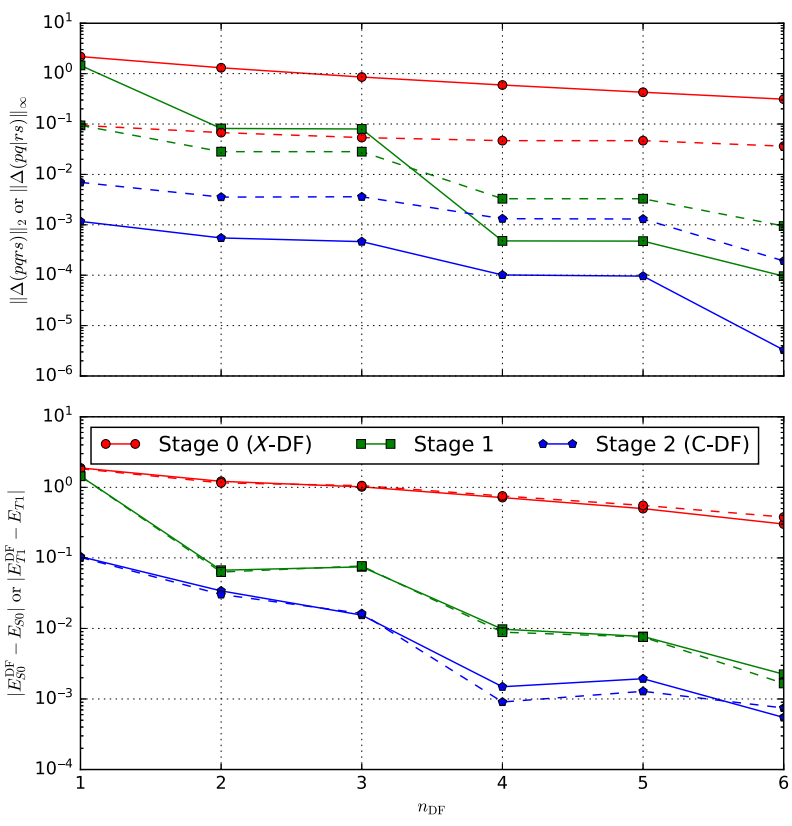

FIG. 3. Error metrics for stage-0 (X-DF), stage-1, and stage2 (C-DF) two-step double factorization approaches for $(K=$ $6)$-double-bond dodecahexene. Minimal $\pi / \pi^{*}$ active space computed from RHF/STO-3G orbitals yielding a (12e, 12o) active space. Top: error characteristics in the ERI tensor, including $\|\Delta(p q \mid r s)\|_{2}$ (solid lines) and $\|\Delta(p q \mid r s)\|_{\infty}$ (dashed lines) as a function of DF auxiliary basis size $n_{\mathrm{DF}}$. Bottom: error characteristics in lowest FCI singlet and triplet state energies $\Delta E_{S 0}$ (solid lines) and $\Delta E_{T 1}$ (dashed lines) as a function of DF auxiliary basis size $n_{\mathrm{DF}}$. In the bottom panel, approximate ERIs are reformed from the DF factorization and then passed into the FCI solver, i.e., the errors shown are for fully variational FCI states computed within the truncated Hamiltonian. 
difficulties in the two-step C-DF fitting procedure. Note also that the apparent even-odd oscillating convergence behavior in Fig. 3 is an artifact of this particular test case, and is not globally representable over other cases that we have encountered. Despite this, the pragmatic error reduction behavior is highly compelling, with $<1 \mathrm{mE}_{h}$ errors achieved by $n_{\mathrm{DF}}=6$, at which point C-DF provides $>100 \times$ error reduction versus $\mathrm{X}-\mathrm{DF}$. In the consideration of stage-1 C-DF versus stage-2 C-DF, full C-DF provides up to approximately $10 \times$ reduction of error for small $n_{\mathrm{DF}}$ in each error characteristic, but the gains appear to narrow somewhat for larger $n_{\mathrm{DF}}$.

Figure 4 shows the error characteristics as a function of system/active space size $K$ for the $(2 \mathrm{Ke}, 2 \mathrm{Ko})$ minimal $\pi / \pi^{*} \mathrm{RHF} / \mathrm{STO}-3 \mathrm{G}$ active spaces of $K$-double-bond linear alkenes ranging from $K=2$ (butadiene) to $K=6$ (dodecahexene), with fixed $n_{\mathrm{DF}}=6$. Note that the $K=1$ case of $(2 \mathrm{e}, 2 \mathrm{o})$ ethylene is not shown, as both X-DF and C-DF
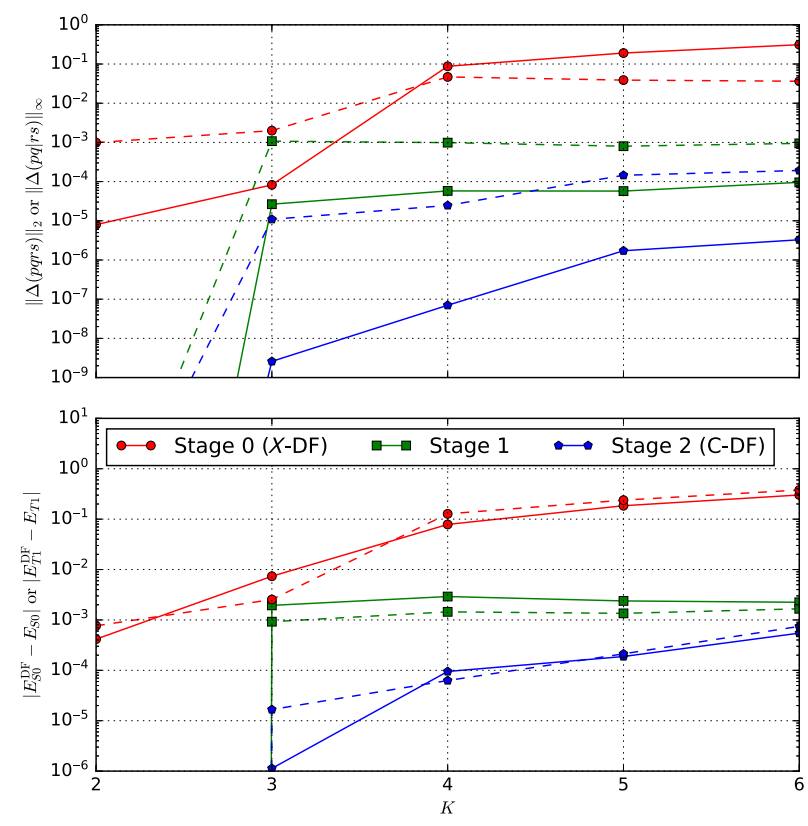

FIG. 4. Error metrics for stage-0 (X-DF), stage-1, and stage-2 (C-DF) two-step double factorization approaches for a constant rank size of $n_{\mathrm{DF}}=6$. Minimal $\pi / \pi^{*}$ active space computed from RHF/STO-3G orbitals for $K=2$ (butadiene) to $K=6$ (dodecahexene) systems with active spaces ranging from $(4 \mathrm{e}, 4 \mathrm{o})$ to (12e, 12o), respectively. Note that $K=1$ (ethylene) with (2e, 20 ) active space is numerically exact with X-DF or C-DF with $n_{\mathrm{DF}}=6$. Top: error characteristics in the ERI tensor, including $\|\Delta(p q \mid r s)\|_{2}$ (solid lines) and $\|\Delta(p q \mid r s)\|_{\infty}$ (dashed lines) as a function of DF auxiliary basis size $n_{\mathrm{DF}}$. Bottom: error characteristics in lowest FCI singlet and triplet state energies $\Delta E_{S 0}$ (solid lines) and $\Delta E_{T 1}$ (dashed lines) as a function of DF auxiliary basis size $n_{\mathrm{DF}}$. In the bottom panel, approximate ERIs are reformed from the DF factorization and then passed into the FCI solver, i.e., the errors shown are for fully variational FCI states computed within the truncated Hamiltonian. are exact with $n_{\mathrm{DF}}=6$ for this case. As in the previous paragraph, we find that trends in the integral error characteristics are largely representative of trends in the FCI state energy characteristics. The X-DF error characteristics [with the possible exception of MAD $\|\Delta(p q \mid r s)\|_{\infty}$ ] show nontrivial size dependence, as expected given the linearscaling expected auxiliary basis of density fitting methods. The C-DF error characteristics are significantly smaller in magnitude (2-3 orders of magnitude of improvement), and also show moderate size dependence. The stage-1 C-DF errors are intermediate between X-DF and C-DF, and show an interesting near-constant scaling characteristic for $K>2$. From this test case, it is not yet clear whether the stage- 1 or stage-2 C-DF is asymptotically constant scaling, linear scaling, or some intermediate (see the conclusion for a discussion in terms of tensor hypercontraction) - any of these might be possible, and further empirical and theoretical analysis is required to resolve this matter. This point notwithstanding, the substantial pragmatic error reduction of $\mathrm{C}-\mathrm{DF}$ is clear from this test case.

Additional insights are provided by considering the same observables for a $(6 \mathrm{e}, 6 \mathrm{o}) \mathrm{RHF} / 6-31 \mathrm{G}^{*}$ active space of bacteriochlorophyll $a$ (BChl $a$ ), with an extensive scan across $n_{\mathrm{DF}}$. Here the moderate active space size allows for tightly converged two-step C-DF fittings, while the globular (e.g., nonlinear) nature of the active space orbitals provides a more challenging test for the C-DF in the regime before pure linear-locality-based asymptotics saturate. Figure 5 shows the error characteristics for this system as a function of $n_{\mathrm{DF}}$ ranging from 1 to 21 . Note that for this $n_{p}=6$ orbital active space, $n_{p}\left(n_{p}+1\right) / 2=21$ unique orbital pairs, so we expect density fitting and X-DF to saturate exactly at $n_{\mathrm{DF}}$. This behavior is indeed observed: the X-DF error characteristics converge roughly monotonically and geometrically, with a sudden drop to double precision exactness at $n_{\mathrm{DF}}=21$. The state energy errors drop below $1 \mathrm{mE}_{h}$ at $n_{\mathrm{DF}}=13$, but the convergence of the state energy errors stagnates somewhat beyond this point. Stage-1 C-DF converges similarly as X-DF (i.e., roughly monotonically and geometrically), albeit with a faster convergence prefactor. The state energy errors drop below $1 \mathrm{mE}_{h}$ at $n_{\mathrm{DF}}=10$ for stage-1 C-DF. Numerical saturation of stage-1 C-DF is achieved for $n_{\mathrm{DF}}=14$. Full C-DF converges much more rapidly, with state energy errors off less than $1 \mathrm{mE}_{h}$ at $n_{\mathrm{DF}}=6$, and onset of numerical saturation at $n_{\mathrm{DF}} \sim 8$. The saturation limits of the various approaches are presumably due to the factorizations having a number of continuous parameters in $Z_{k l}^{t}$ and/or $X_{k l}^{t}$ that exceed the number of degrees of freedom in the ERI tensor. This numerical saturation should not be relied on for larger active spaces, as it will asymptotically require a quartic number of parameters for mediumsized, globular systems. Overall, these data are not yet sufficient to firmly establish the asymptotic scalings of 

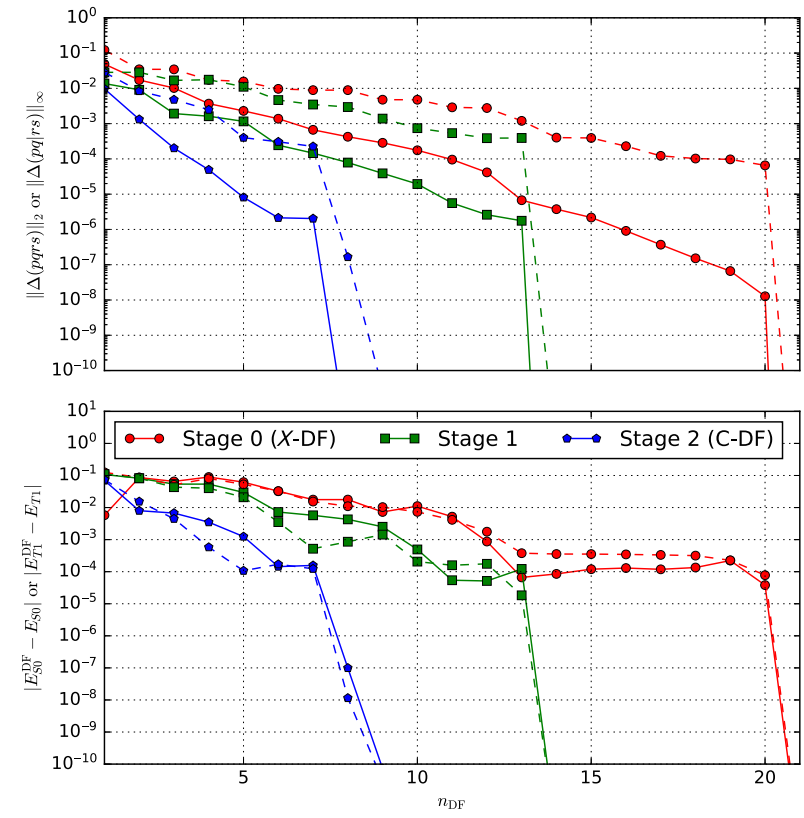

FIG. 5. Error metrics for stage-0 (X-DF), stage-1, and stage2 (C-DF) two-step double factorization approaches for $\mathrm{BChl}$ $a$. Valence active space computed from RHF/6-31G* orbitals, yielding a (6e, 6o) active space. Top: error characteristics in the ERI tensor, including $\|\Delta(p q \mid r s)\|_{2}$ (solid lines) and $\|\Delta(p q \mid r s)\|_{\infty}$ (dashed lines) as a function of DF auxiliary basis size $n_{\mathrm{DF}}$. Bottom: error characteristics in lowest FCI singlet and triplet state energies $\Delta E_{S 0}$ (solid lines) and $\Delta E_{T 1}$ (dashed lines) as a function of DF auxiliary basis size $n_{\mathrm{DF}}$. In the bottom panel, approximate ERIs are reformed from the DF factorization and then passed into the FCI solver, i.e., the errors shown are for fully variational FCI states computed within the truncated Hamiltonian.

C-DF. However, the substantial pragmatic reduction in $n_{\mathrm{DF}}$ from 13 (X-DF) to 6 (C-DF) to achieve chemical accuracy in total state energies is a compelling numerical finding.

\section{Two-step C-DF iterative convergence characteristics}

It should be noted that $\mathrm{C}-\mathrm{DF}$ is not a panacea in the sense that (1) often a very large number of L-BFGS optimization epochs are required to obtain substantive convergence and (2) in some cases, substantive numerical convergence is not obtained at all within $10^{4} \mathrm{~L}-\mathrm{BFGS}$ epochs. This is highlighted in Fig. 6, which shows the convergence characteristics of the two-step C-DF fitting procedure as a function of L-BFGS iteration for the representative case of $\mathrm{BChl} a$ with $(6 \mathrm{e}, 6 \mathrm{o})$ valence active space of $\mathrm{RHF} / 6-31 \mathrm{G}^{*}$ orbitals. Here we see three paradigms of convergence behavior. (1) At small $n_{\mathrm{DF}}$ of approximately $1-3$, very tight convergence of the gradient can be obtained to a fixed point with a minimal but nonzero objective function $O$. (2) At intermediate $n_{\mathrm{DF}}$ of approximately $4-7$, the objective function $O$ is significantly reduced by the two-step C-DF iterative procedure, but a tightly converged gradient is not
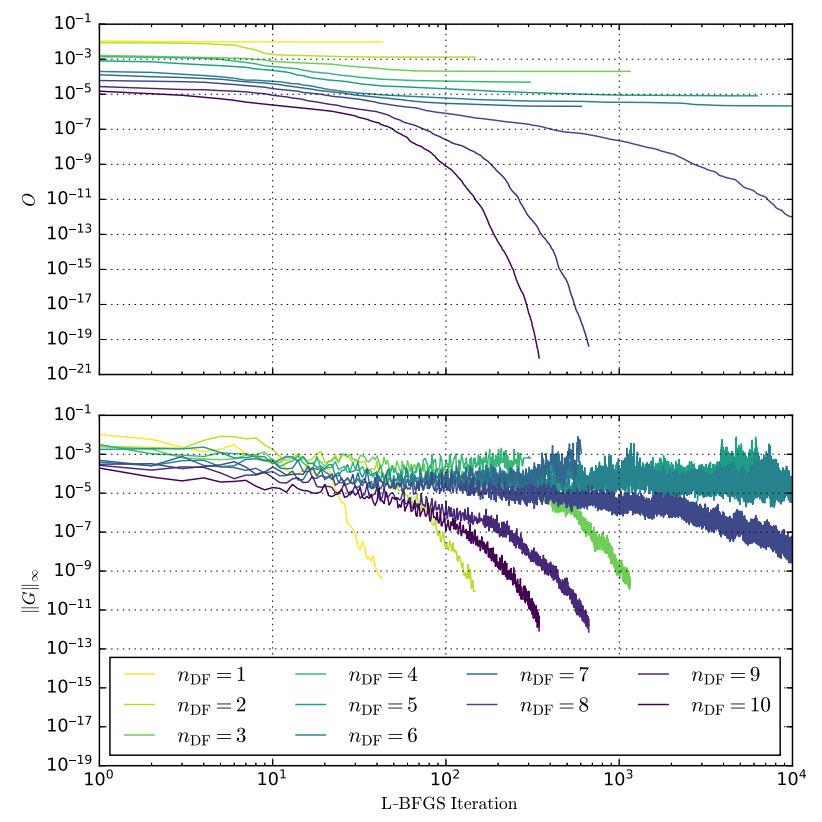

FIG. 6. Convergence characteristics of the two-step C-DF fitting procedure as a function of L-BFGS iteration for the case of BChl $a$ with $(6 \mathrm{e}, 6 \mathrm{o})$ valence active space of RHF/6-31G* orbitals. Top: convergence behavior of the C-DF objective function $O$. Bottom: convergence of the infinity norm of the C-DF objective function gradient $\vec{G} \equiv \partial O / \partial X_{p k}^{t}$.

obtained in the $10^{4}$ iterations allowed here. (3) At large $n_{\mathrm{DF}}$ of approximately 8-9, very tight convergence of the gradient can be obtained to a fixed point with a numerically zero objective function $O$. This indicates that additional work should be done to improve the convergence behavior of the two-step C-DF optimization procedure, particularly for intermediate sizes of $n_{\mathrm{DF}}$. This issue aside, C-DF seems to provide remarkable improvement over X-DF in many cases, particularly including the highly important case of small $n_{\mathrm{DF}} \mathrm{DF}$ rank expansion.

We use C-DF for all QFD cases discussed later in this work.

\section{B. Quantum filter diagonalization}

Quantum filter diagonalization is a technique for approximating eigenpairs of a Hamiltonian operator $\hat{H}$ $[3,4]$. It makes use of a set of time-propagated states,

$$
\left|\Psi_{m}\right\rangle=e^{-i \Delta t m \hat{H}}\left|\Psi_{0}\right\rangle=\hat{U}_{\Delta t}^{m}\left|\Psi_{0}\right\rangle,
$$

as a variational basis for approximate diagonalization of $\hat{H}$. In Eq. (31), $\hat{U}_{\Delta t}$ is the time-evolution operator under the Hamiltonian $\hat{H}$ for time $\Delta t, m=0, \ldots, n_{\mathrm{QFD}}-1$ is an integer number, and $\left|\Psi_{0}\right\rangle$ is a "guess" quantum state determined by classical preprocessing that can be prepared efficiently by a quantum circuit, such as a Slater determinant. A straightforward variant of the method allows for 


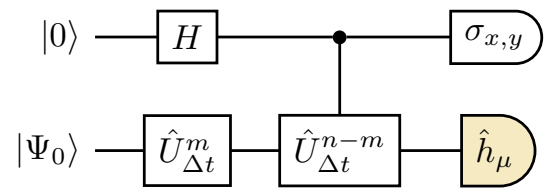

FIG. 7. Quantum circuits for measuring the QFD matrix element $H_{m, n}$. Here $\hat{h}_{\mu}$ denotes a term of the Hamiltonian, and $\hat{U}_{\Delta t}$ the circuit implementing time evolution under the Hamiltonian for a time step $\Delta t$. The overlap matrix element $S_{m, n}$ is measured replacing $\hat{h}_{\mu}$ with the identity operator.

the use of a basis of multiple nonredundant guess states [3], but in this work, we always use a single guess state. Approximations for the eigenstates of $\hat{H}$ are constructed as linear combinations of the basis vectors,

$$
\left|\Phi_{I}\right\rangle=\sum_{m=0}^{n_{\mathrm{QFD}}-1} c_{m I}\left|\Psi_{m}\right\rangle,
$$

where the coefficients are determined by classically solving the generalized eigenvalue equation $H c_{I}=\varepsilon_{I} S c_{I}$, where

$$
S_{m, n}=\left\langle\Psi_{m} \mid \Psi_{n}\right\rangle, \quad H_{m, n}=\left\langle\Psi_{m}|\hat{H}| \Psi_{n}\right\rangle .
$$

The overlap and Hamiltonian matrices $S$ and $H$ are computed using a set of extended Hadamard quantum circuits [30] with a single ancilla, illustrated in Fig. 7.

Indeed, it can be easily shown that

$$
\left\langle\Psi_{m}\left|\hat{h}_{\mu}\right| \Psi_{n}\right\rangle=\left\langle\chi\left|X \otimes \hat{h}_{\mu}\right| \chi\right\rangle+i\left\langle\chi\left|Y \otimes \hat{h}_{\mu}\right| \chi\right\rangle,
$$

where

$$
|\chi\rangle=\frac{|0\rangle \otimes \hat{U}_{\Delta t}^{m}\left|\Psi_{0}\right\rangle+|1\rangle \otimes \hat{U}_{\Delta t}^{n-m}\left|\Psi_{0}\right\rangle}{\sqrt{2}}
$$

is the output state of the quantum circuit in Fig. 7.

In general, the exact time-evolution operator $\hat{U}_{\Delta t}$ is not known. On a digital quantum computer, it can be approximated with accuracy $\delta$ at cost up to $\operatorname{poly}\left(n_{p}, \Delta t, \delta^{-1}\right)$ [2]. A prominent example is the primitive Trotter approximation

$$
\hat{U}_{\Delta t} \simeq \prod_{\mu} e^{-i \Delta t \hat{h}_{\mu}}, \quad \hat{H}=\sum_{\mu} \hat{h}_{\mu},
$$

where, for each operator $\hat{h}_{\mu}$, the quantum circuits implementing $e^{-i \Delta t \hat{h}_{\mu}}$ are known.

In this work, we introduce primitive Trotter approximation (36) into the definition of the basis vectors $\left|\Psi_{m}\right\rangle$. Within such an approximation, the Toeplitz property $S_{m, n}=S_{0, n-m}, H_{m, n}=H_{0, n-m}$ enjoyed by the exact overlap and Hamiltonian matrix elements (32) is lost, so that the number of quantum circuits to be evaluated scales as $\mathcal{O}\left(n_{\mathrm{QFD}}{ }^{2}\right)$. Nevertheless, the QFD algorithm retains a number of desirable features, especially numerical stability and the structure of a variational wavefunction ansatz [3].

In the next section, we explore how the C-DF representation of the electron repulsion integral can be used to economize QFD circuits, and the measurement of QFD matrix elements.

\section{Circuit reduction strategies}

To describe the circuit reductions allowed by the combination of QFD and DF, we express the Hamiltonian as in Eq. (9),

$$
\begin{aligned}
\hat{H}= & E_{\mathrm{Ext}}+\sum_{k} f_{k k}^{0} \hat{E}_{k k}^{+}(0)+\sum_{t} \sum_{k l} \frac{Z_{k l}^{t}}{2} \hat{E}_{k k}^{+}(t) \hat{E}_{l l}^{+}(t) \\
= & E_{\mathrm{Ext}}+\hat{G}_{0 \leftarrow \mathrm{HF}}\left[\sum_{k \sigma} f_{k k}^{0} \hat{n}_{k, \sigma}\right] \hat{G}_{\mathrm{HF} \leftarrow 0} \\
& +\sum_{t} \hat{G}_{t \leftarrow \mathrm{HF}}\left[\sum_{k l, \sigma \tau} \frac{Z_{k l}^{t}}{2} \hat{n}_{k, \sigma} \hat{n}_{l, \tau}\right] \hat{G}_{\mathrm{HF} \leftarrow t .}
\end{aligned}
$$

Here, $\hat{G}_{\mathrm{HF} \leftarrow l}$ denotes a unitary transforming from the Hartree-Fock basis to the eigenbasis of the $l$ th term of the Hamiltonian. The index $l=0$ represents the one-body term, and the indices $l=1, \ldots, n_{\mathrm{DF}}$ represent the terms of the double-factorized Hamiltonian.

Under the Jordan-Wigner mapping, number operators $\hat{n}_{k, \sigma}$ and products of number operators $\hat{n}_{k, \sigma} \hat{n}_{l, \tau}$ take the form

$$
\begin{aligned}
\hat{n}_{k, \sigma} & =\frac{\left(1-Z_{k, \sigma}\right)}{2}, \\
\hat{n}_{k, \sigma} \hat{n}_{l, \tau} & =\frac{\left(1-Z_{k, \sigma}\right)}{2} \frac{\left(1-Z_{l, \tau}\right)}{2} .
\end{aligned}
$$

As seen, products of number operators contain terms that are linear in $Z_{k, \sigma}$. We also note here a slight abuse of notation where $Z_{l k}^{t}$ is used to denote the core tensors and $Z_{k, \sigma}$ is used to denote the Pauli- $Z$ operator. The notation is differentiated by the use of a superscript for the core tensors throughout the text. Our goal is to reorganize the Hamiltonian into a new one-body part and a set of two-body factors involving only products of the form $Z_{k, \sigma} Z_{l, \tau}$. To this end, we introduce the operators $\hat{z}_{k, \sigma}=1-2 \hat{n}_{k, \sigma}$ and recall that $\hat{z}_{k, \sigma}^{2}=1$; we can readily recast Eq. (9) in the form

$$
\begin{aligned}
\hat{H}= & E_{\mathrm{Ext}}^{\prime}+\hat{G}_{0 \leftarrow \mathrm{HF}}^{\prime}\left[\sum_{k \sigma} f_{k k}^{\prime} \hat{z}_{k, \sigma}\right] \hat{G}_{\mathrm{HF} \leftarrow 0}^{\prime} \\
& +\sum_{t} \hat{G}_{t \leftarrow \mathrm{HF}}\left[\sum_{k l, \sigma \tau}^{*} \frac{Z_{k l}^{t}}{8} \hat{z}_{k, \sigma} \hat{z}_{l, \tau}\right] \hat{G}_{\mathrm{HF} \leftarrow t},
\end{aligned}
$$


where the asterisk denotes summation over strings $k l, \sigma \tau$ with $k \neq l$, or $k=l$ and $\sigma \neq \tau$, and primes denote a simple redefinition of the Hamiltonian coefficients and of the unitary transforming from the Hartree-Fock basis to the eigenbasis of the one-body part of the Hamiltonian.

In this form, time evolution under the Hamiltonian for a time step $\Delta t$ can be approximated by

$$
\hat{U}_{\Delta t} \simeq e^{-i \Delta t E_{\mathrm{Ext}}^{\prime}} \hat{G}_{n_{\mathrm{DF}} \leftarrow \mathrm{HF}}\left[\prod_{t} \hat{V}_{2 b, t} \hat{G}_{t-1 \leftarrow t}\right] \hat{V}_{1 b} \hat{G}_{\mathrm{HF} \leftarrow 0}^{\prime}
$$

where

$$
\begin{aligned}
\hat{V}_{1 b} & =\prod_{k, \sigma} e^{-i \Delta t f_{k k}^{\prime} \hat{z}_{k, \sigma},} \\
\hat{V}_{2 b, t} & =\prod_{k l, \sigma \tau}^{*} e^{-i \Delta t Z_{k l}^{t} \hat{z}_{k, \sigma} \hat{z}_{l, \tau} / 8},
\end{aligned}
$$

and $\hat{G}_{t-1 \leftarrow t}=\hat{G}_{\mathrm{HF} \leftarrow t} \hat{G}_{\mathrm{t}-1 \leftarrow \mathrm{HF}}$.

In this form, each of the change-of-basis unitaries $\hat{G}$ factors in two identical parts, acting on spin-up and spin-down spin orbitals, respectively. Such parts can be compiled into networks of Givens rotations, which can in turn be represented with one- and two-qubit gates, as discussed in the Appendix A 1. Furthermore, in the Jordan-Wigner representation, evolution under the one-body part of the Hamiltonian can be implemented by a network of single-qubit $Z$ rotations, and each of the terms $\exp \left(-i \Delta t Z_{k l}^{t} \hat{z}_{k, \sigma} \hat{z}_{l, \tau}\right)$ can be implemented with two controlled-NOT (CNOT) and single-qubit $Z$ rotations, as discussed in the Appendix A 1.

The quantum circuit implementing the controlled version of the unitary transformation Eq. (40) is shown in Fig. 8. It is useful to observe that unitaries $\hat{G}$ multiply to the identity and thus, as seen in Fig. 8, they need not be controlled. In the Jordan-Wigner representation, each controlled $\exp \left(-i \Delta t f_{k k}^{\prime} \hat{z}_{k, \sigma}\right)$ can be constructed with two CNOT and two single-qubit $Z$ rotations, whereas each controlled $\exp \left(-i \Delta t Z_{k l}^{t} \hat{z}_{k, \sigma} \hat{z}_{l, \tau}\right)$ can be constructed with four CNOT and two two-qubit $Z Z$ rotations.

\section{Error mitigation strategies}

\section{Postselection}

The structure of the Hamiltonian highlighted in Eq. (40) allows for a simple scheme for measurement postselection, based on enforcing the correct numbers $N_{\alpha}, N_{\beta}$ of particles for each spin species [19]. The $\hat{h}_{\mu}$ referenced in Fig. 7 are

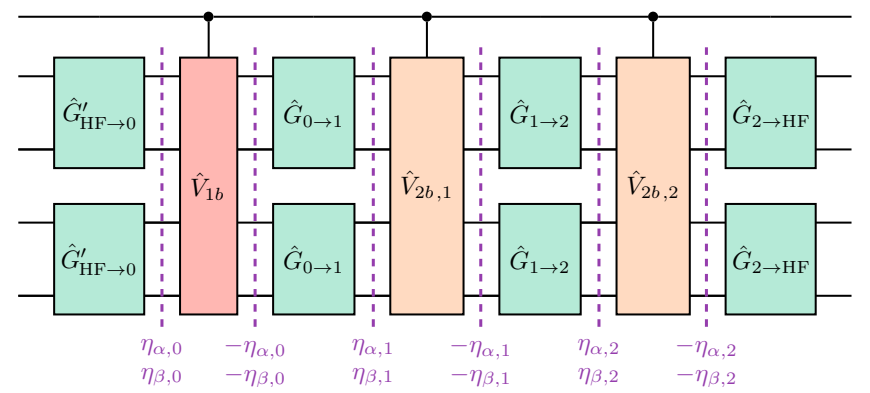

FIG. 8. Quantum circuit implementing time evolution under the DF Hamiltonian for a time step $\Delta t$. Green, red, and orange blocks denote change-of-basis unitaries and evolutions under diagonal one-body and diagonal two-body operators, respectively, and violet lines denote echo unitaries parametrized by angles $\eta_{k}$.

represented by our operators

$$
\begin{aligned}
\hat{h}_{0} & =\sum_{k \sigma} f_{k k}^{\prime} \hat{z}_{k, \sigma}, \\
\hat{h}_{t} & =\sum_{k l, \sigma \tau}^{*} \frac{Z_{k l}^{t}}{8} \hat{z}_{k, \sigma} \hat{z}_{l, \tau}, \quad t=1, \ldots, n_{\mathrm{DF}} .
\end{aligned}
$$

As shown in Fig. 9, we can measure each of these operators in their respective diagonal basis by first applying an appropriate unitary transformation, and then making a projective measurement in the computational basis.

The benefit of this measurement scheme is that $N_{\alpha}$ and $N_{\beta}$ are simultaneously diagonalized in each basis. This means that $N_{\alpha}$ and $N_{\beta}$ can be extracted from each measurement shot of each operator in our Hamiltonian. From this information we can discard any shots that return an incorrect number of particles of each spin polarization.

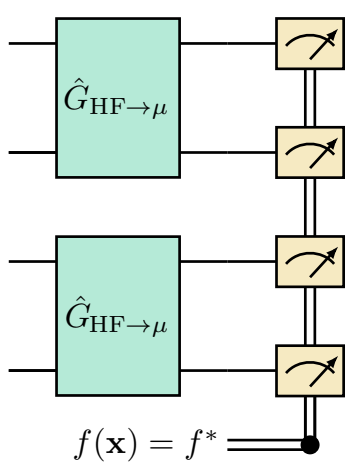

FIG. 9. Quantum circuit implementing the measurement of a term of the C-DF Hamiltonian. Green blocks denote change-ofbasis unitaries, and yellow meters denote measurement of single qubits. Only measurement outcomes $\mathbf{x}$ with the correct number of spin-up and spin-down particles are retained in the postselection algorithm. 


\section{Echo sequencing}

In Ref [31] the authors introduced the idea of echo sequencing each full Trotter step by the inherent symmetries of the Hamiltonian. A standard simulation

$$
e^{-i t \hat{H}} \approx \prod_{j} \hat{U}_{\Delta t}
$$

is replaced with a symmetry-protected one,

$$
e^{-i t \hat{H}} \approx \prod_{j} \hat{C}_{j}^{\dagger} \hat{U}_{\Delta t} \hat{C}_{j}, \quad\left[\hat{C}_{j}, \hat{H}\right]=0
$$

resulting in a reduction of the second-order Trotter error.

In this work, the Trotter step takes the form

$$
U_{\text {target }}(\Delta t)=\prod_{\mu} e^{-i \Delta t \hat{h}_{\mu}},
$$

and, to first order in the Schrödinger representation, time evolution acts as

$$
\rho(\Delta t)=\rho(0)-i \Delta t \sum_{\mu}\left[\rho(0), \hat{h}_{\mu}\right]
$$

Here, $\left[\hat{N}_{\alpha / \beta}, \hat{h}_{\mu}\right]=0$, which provides the opportunity to simultaneously echo both the $\alpha$ and $\beta$ spin sectors for each term $\exp \left[-i \Delta t \hat{h}_{\mu}\right]$. We assume that gate errors exhibited on the hardware induce an additional coherent bias term $\hat{V}_{\mu}$ for each operator $\hat{h}_{\mu}$. When implementing time evolution, each block $\exp \left(-i \Delta t \hat{h}_{\mu}\right)$ takes the form

$$
U_{\exp }(\Delta t)=\prod_{\mu} e^{-i \Delta t\left(\hat{h}_{\mu}+\hat{V}_{\mu}\right)}
$$

then, when expanding to first order in the Heisenberg representation, we now have

$$
\rho(\Delta t)=\rho(0)-i \Delta t \sum_{\mu}\left[\rho(0), \hat{h}_{\mu}+\hat{V}_{\mu}\right]
$$

On the other hand, if we echo with both $\hat{N}_{\alpha}$ and $\hat{N}_{\beta}$ for each term $\exp \left[-i \Delta t \hat{h}_{\mu}\right]$, this leaves

$$
\begin{aligned}
U_{\text {echo }}(\Delta t) & =\prod_{\mu} \hat{C}_{\mu} e^{-i \Delta t\left(\hat{h}_{\mu}+\hat{V}_{\mu}\right)} \hat{C}_{\mu}^{\dagger}, \\
\hat{C}_{\mu} & =e^{i \eta_{\alpha, \mu} \hat{N}_{\alpha}} e^{i \eta_{\beta, \mu} \hat{N}_{\beta}},
\end{aligned}
$$

where $\eta_{\alpha / \beta, \mu}$ is a random phase with uniform distribution in the interval $[0,2 \pi]$. The average of the first-order expansion over the random phases is

$$
\bar{\rho}(\Delta t)=\rho(0)-i \Delta t \sum_{\mu}\left[\rho(0), \hat{h}_{\mu}+P_{N_{\alpha}, N_{\beta}}^{\dagger} \hat{V}_{\mu} P_{N_{\alpha}, N_{\beta}}\right],
$$

where $P_{N_{\alpha}, N_{\beta}}$ is the projection operator on the proper symmetry sector.

Therefore, this echo-sequencing scheme results in the suppression of error terms that couple different symmetry sectors together $[32,33]$. The ability to conduct $L+1$ echoes per Trotter step instead of a single echo should result in a higher capacity for error mitigation, especially when $L$ is large.

Under the Jordan-Wigner mapping, the echo terms for each spin species is simply a product of single-qubit $\mathbf{Z}$ rotations, which can be implemented with minimal overhead. The echo scheme can be implemented under the parity mapping as well, but this requires applying the operators $\exp \left[-i \eta\left(Z_{0}+Z_{0} Z_{1}+Z_{1} Z_{2}+\cdots+Z_{N-1} Z_{N}\right)\right]$, and thus involves a greater overhead.

\section{RESULTS AND DISCUSSION}

The strategy for the calculations performed in this work involved initial preprocessing by classical quantum chemistry codes on conventional computers, to generate optimized Hartree-Fock orbitals and matrix elements of the Hamiltonian in active spaces of 2, 4, or 6 orbitals, prior to performing computations with quantum simulators or devices.

The chemical species studied in this work are shown in Fig. 10 at geometries listed in Appendix A. These medium-sized photoactive molecules have interesting photochemistry dictated by a handful of active orbitals. These systems provide realistic test cases with active spaces of (2e, 2e) and upwards. The Hamiltonian is constructed from an active space of restricted Hartree-Fock (RHF) singlet spatial orbitals, computed via the Lightspeed/TeraChem package, for all computations performed herein.

Quantum calculations are performed using IBM's opensource PYTHON library for quantum computing, Qiskit (a)

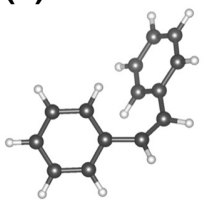

(b)

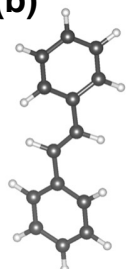

(c)

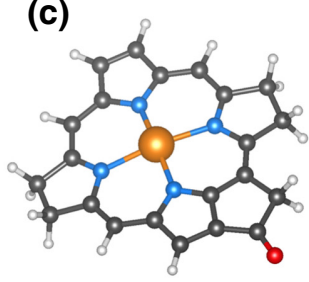

(d)

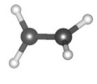

FIG. 10. Molecular species studied in the present work: cisand trans-stilbene, bacteriochlorophyll $a(\mathrm{BChl} a)$, and ethylene [left to right, (a) to (d)]. 
[34]. Qiskit provides tools for various tasks such as creating quantum circuits, performing simulations, and computations on quantum devices. We ran our experiments on both the statevector and qasm simulators in Qiskit, and performed hardware experiments on 16- and 28-qubit devices available through IBM Quantum Experience with a quantum volume [35] of 32, namely, ibmq guadalupe, ibmq_montreal, and ibmq_mumbai [36].

\section{A. Classical simulations}

We begin our analysis by computing, in Fig. 11, the exact singlet-triplet and singlet-singlet gaps, $\Delta E_{S_{0} T_{1}}$ and $\Delta E_{S_{0} S_{1}}$, respectively, for stilbene and BChl $a$. We use the exact and doubly factorized Hamiltonian, with $n_{\mathrm{DF}}=$ $1,2,3,4$ layers of tensors, with the purpose of assessing the accuracy of the double factorization. As seen, for active spaces of $M=2$ orbitals, $n_{\mathrm{DF}}=2$ layers are sufficient to obtain an exact representation of the electron repulsion integral, and thus exact gaps. For $M=4,6$ orbitals, on the other hand, $n_{\mathrm{DF}}=4$ layers are needed to achieve accuracy of the order of a milli-Hartree.

In Figs. 7, 8, and 9 we perform exact simulations of the QFD algorithm for cis-stilbene, trans-stilbene, and BChl $a$, respectively, using active spaces of $M=2$ to 6 orbitals. As naturally expected, and in accordance with the observations of Fig. 11, increasing the number $n_{\mathrm{DF}}$ of layers in the doubly factorized representation of the Hamiltonian brings energy differences systematically closer to exact values for the full Hamiltonian. Indeed, with $n_{\mathrm{DF}}=1$, deviations between singlet-triplet and singlet-singlet gaps of the exact and doubly factorized Hamiltonian are of the order of 2 to $40 \mathrm{mHa}$, and decrease as $n_{\mathrm{DF}}$ increases. For all species considered here, the singlet-singlet gap from the doubly factorized Hamiltonian is closer to the exact value than the singlet-singlet gap, indicating that the latter quantity is more sensitive to approximations in the electron repulsion integral.

For cis- and trans-stilbene, increasing the number $n_{\mathrm{QFD}}$ of time evolution steps in the QFD algorithm has little effect on energy differences. A different behavior is seen in Fig. 9 for $\mathrm{BChl} a$, where energy differences show variations of the order of a few milli-Hartree as $n_{\mathrm{QFD}}$ varies. The difference between the behavior of stilbene and $\mathrm{BChl}$ $a$ stems from the different point group symmetries of the geometries studied here $\left(C_{2}\right.$ and $C_{1}$ for stilbene and $\mathrm{BChl}$ $a$, respectively). Such a difference is particularly visible in the case of active spaces with $M=2$ orbitals: for $C_{2}-$ symmetric species, the ground and $S_{2}$ excited states lie in the $A$ irrep of the $C_{2}$ symmetry group, and are thus automatically orthogonal to the triplet and $S_{1}$ excited states, which instead lie in the $B$ irrep. Therefore, a single step of time evolution applied to the Hartree-Fock state ( $A$ irrep) or to a configuration with a single HOMO-LUMO excitation ( $B$ irrep) is sufficient to completely span the subspaces

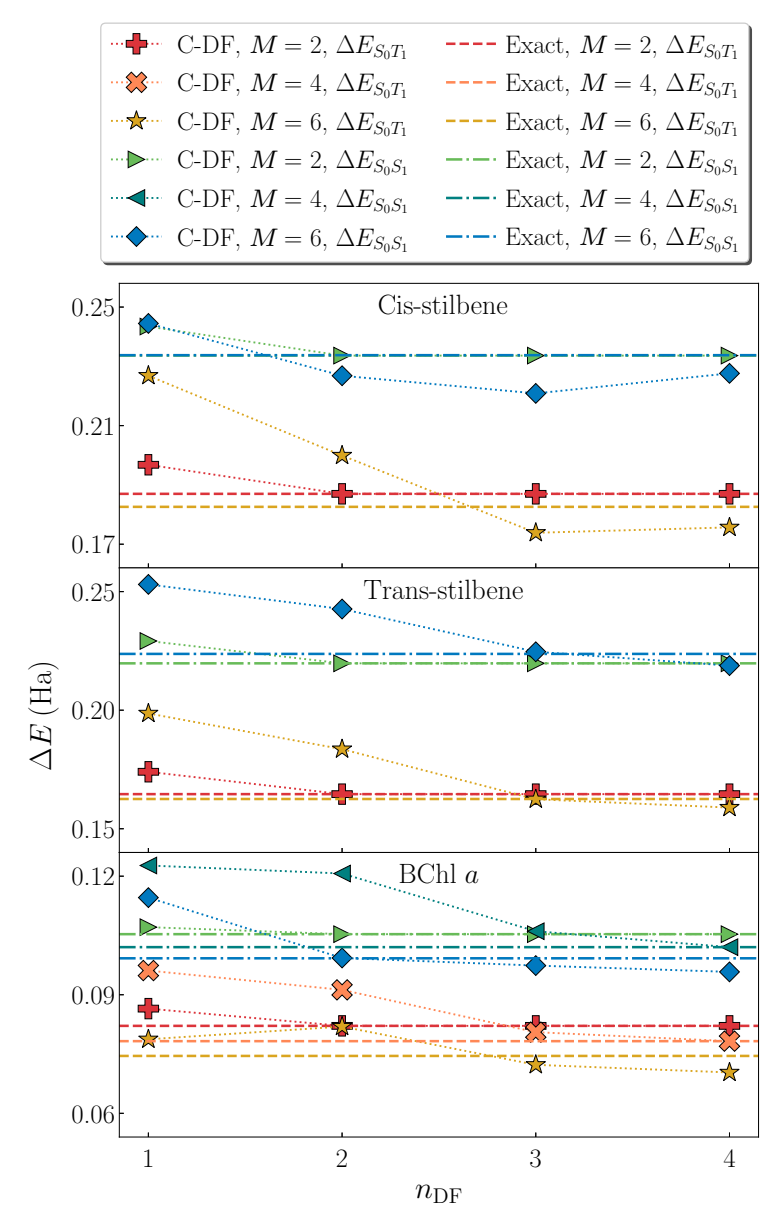

FIG. 11. Singlet-triplet $\Delta E_{S_{0} T_{1}}$ and singlet-singlet $\Delta E_{S_{0} S_{1}}$ gap from the exact (dashed, dash-dot lines) and the C-DF Hamiltonian (markers) with $n_{\mathrm{DF}}=1,2,3,4$ layers for cis-stilbene, trans-stilbene, and BChl $a$ (top to bottom) in active spaces of $M=2$ (red and green symbols), 4 (orange and teal symbols), and 6 (yellow and blue symbols) orbitals.

of $A$ and $B$ symmetric wavefunctions. In the more general case of BChl $a$, where such a simplification does not occur, two time evolution steps are needed.

In Fig. 12, we perform exact simulations of the QFD algorithm for cis-stilbene, trans-stilbene, and $\mathrm{BChl} a$, respectively, using active spaces of $M=6$ orbitals. As seen, and in accordance with the observations of Fig. 11, increasing the number $n_{\mathrm{DF}}$ of layers in the doubly factorized representation of the Hamiltonian brings energy differences closer to exact values for the full Hamiltonian (faint shades of blue and red). Increasing $n_{\mathrm{DF}}$ is more critical to converge energy differences than $n_{\mathrm{QDF}}$. It should also be noted that, when $n_{\mathrm{DF}}$ is small, C-DF can underestimate energy differences. This is demonstrated in Appendix A 2 with Fig. 19.

Increasing the number of QFD steps $n_{\mathrm{QFD}}$ affects energy differences on a milli-Hartree scale. This effect is documented in Fig. 13, using BChl $a$ as an example with $M=6$ 


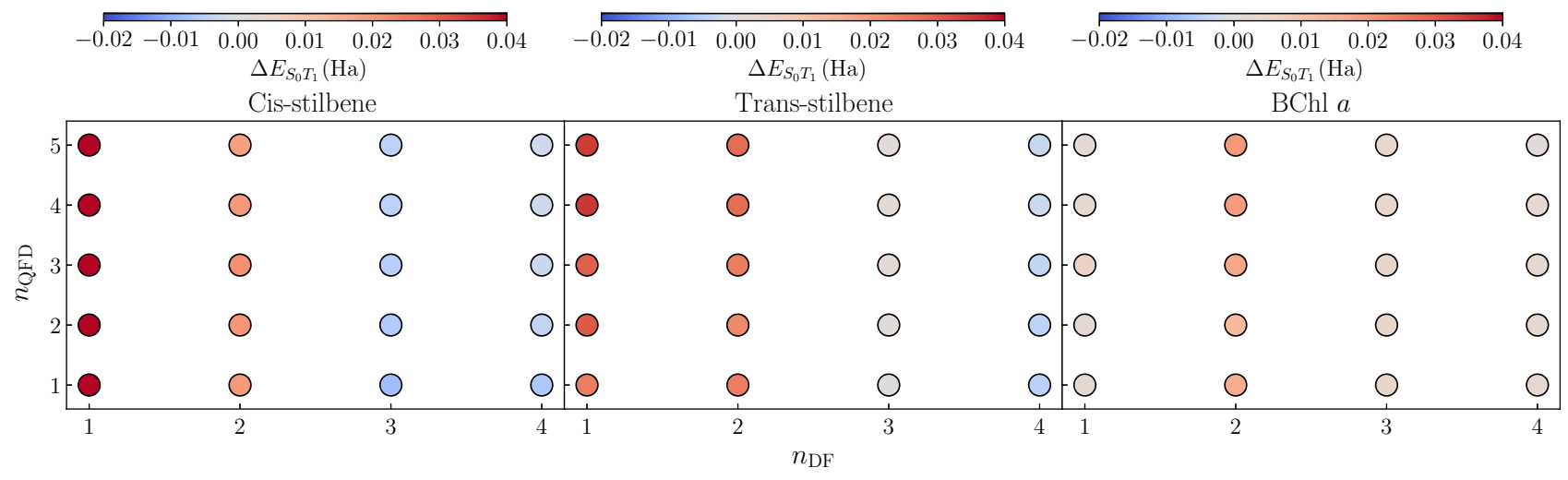

FIG. 12. Deviation between QFD and FCI singlet-triplet $\Delta E_{S_{0} T_{1}}$ gap as a function of the number of QFD steps ( $\left.n_{\mathrm{QFD}}\right)$ and C-DF layers $\left(n_{\mathrm{DF}}\right)$ for cis-stilbene, trans-stibene, and BChl $a$ (left to right) using active spaces of $M=6$ orbitals. Calculations use a time step of $\Delta t=20 /\left(n_{\mathrm{QFD}} n_{\mathrm{DF}}\right) \mathrm{Ha}^{-1}$.

orbitals. We see that there is a clear bias coming from the QFD method compared to $\mathrm{FCI}_{n_{\mathrm{DF}}}$. As seen, when $n_{\mathrm{QFD}}$ is increased, the energies decrease by a few milli-Hartree. Such a decrease is naturally expected, given the variational nature of QFD, and the fact that increasing $n_{\text {QFD }}$ means projecting the Schrödinger equation onto increasingly larger variational subspaces. The slow convergence is likely due to the choice of time step. When referring back to Fig. 11, singlet-triplet gaps have weaker dependence on $n_{\mathrm{QFD}}$ than total singlet and triplet energies, due to cancelation of errors. Direct comparisons between the

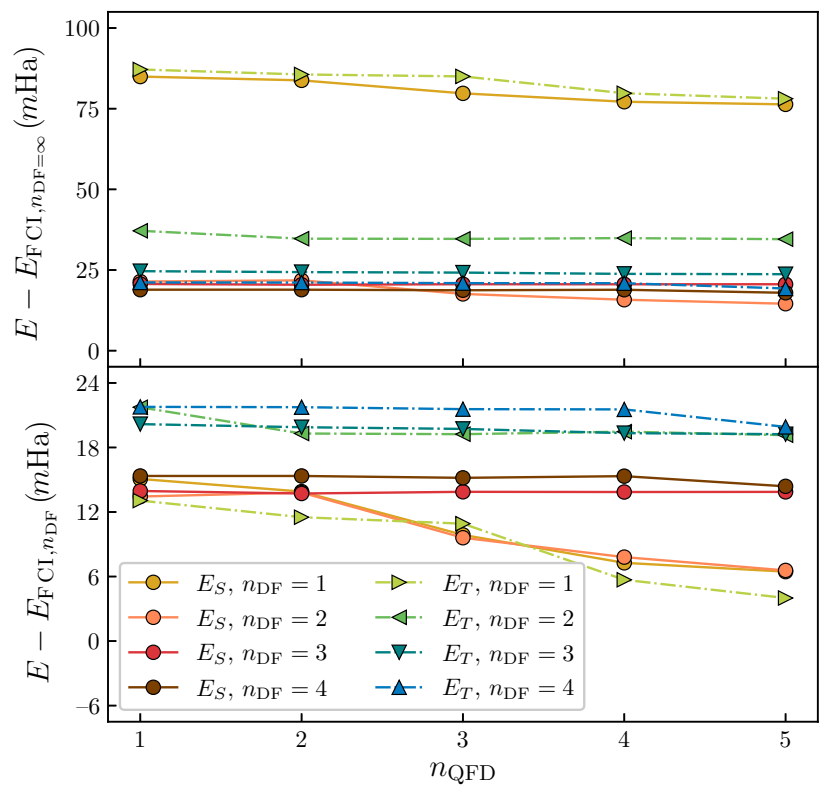

FIG. 13. Singlet and triplet (cool, warm colors) energies for BChl $a$, as a function of $n_{\mathrm{QFD}}$, and for several values of $n_{\mathrm{DF}}$, compared against results from the exact electron repulsion integral (top) and the C-DF electron repulsion integral with $n_{\mathrm{DF}}$ layers (bottom) using active spaces of $M=6$ orbitals.
$\mathrm{X}$-DF and C-DF methods of $\mathrm{FCI}_{n_{\mathrm{DF}}}$ as a function of $n_{D F}$ can be found in Appendix A 2.

In Fig. 14, we perform classical emulations of the QFD algorithm, using a simulator (qasm) that accounts for statistical uncertainties affecting results of quantum mechanical measurements, and incorporate the effect of various decoherence phenomena through noise models. Errors arising from decoherence are mitigated with a combination of the postselection and echo-sequencing techniques described in Sec. II. Deviations between exact and computed energies are listed in Table I in Appendix B.

The energies of ground $S_{0}$ and $T_{1}, S_{1}$, and $S_{2}$ excited states are found to be in agreement with exact results across torsion of the $\mathrm{C}-\mathrm{C}$ bond. The effect of postselection and echo-sequencing are illustrated in the right part of the figure: raw (i.e., unmitigated) data have deviations from exact results and statistical uncertainties of the order of a few tens of milli-Hartrees. Upon postselection, both deviations and statistical uncertainties decrease to order 10 mHa.

A similar effect is seen when the echo-sequencing technique is applied, which arises because of echo sampling, and because averaging results over $n_{\text {echo }}$ calculations reduces statistical uncertainties by a factor $n_{\text {echo }}{ }^{-1 / 2}$. Finally, the combination of postselection and echo sequencing is seen to reduce statistical uncertainties to 1-2 mHa, and deviations between computed and exact results are statistically compatible with zero within such statistical uncertainties.

\section{B. Hardware experiments}

All of the hardware experiments are run with a HOMOLUMO active space, with $n_{\mathrm{DF}}=1$, and a single time step. We also compare the calculations performed using parity mapping and two-qubit reduction (P2QR) and Jordan Wigner (JW) that require a total of three and five qubits, 

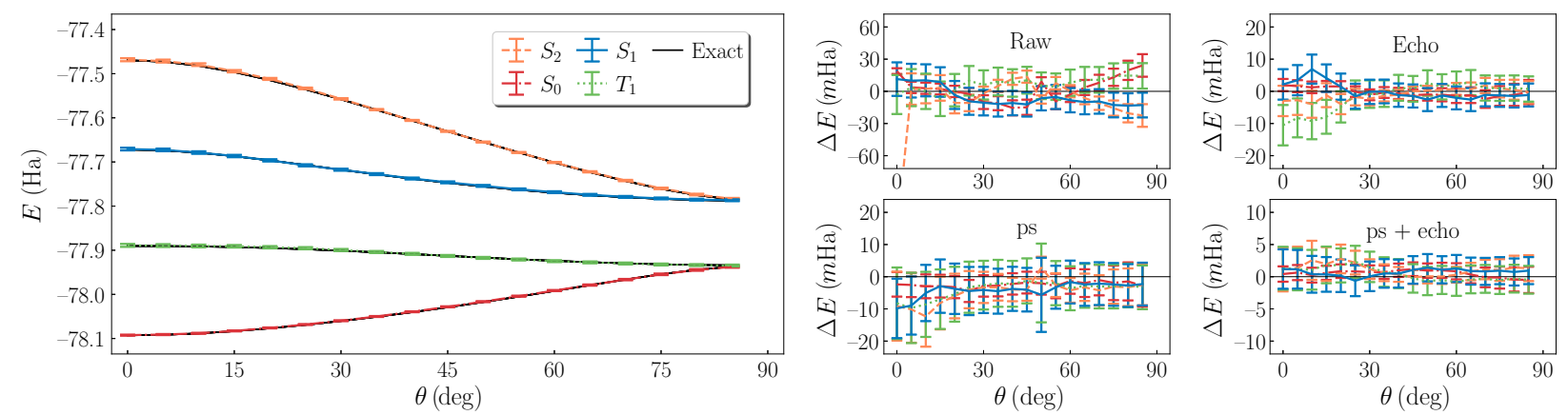

FIG. 14. Left: exact (black) and QFD (colored) energies as a function of torsion angle for ethylene, using a $M=2$ orbital active space, $n_{\mathrm{DF}}=n_{\mathrm{QFD}}=1$, and $\Delta t=0.1 \mathrm{Ha}^{-1}$. Postselection and echo sequencing with $n_{\text {echo }}=10$ samples are used, and calculations are carried out on a classical simulator with noise model from ibmq montreal. Right: differences between QFD and exact energies for calculations with no error mitigation (raw), postselection (ps), echo sequencing (echo), and postselection and echo sequencing (ps + echo).

respectively. The restriction to a single time step and single C-DF factor is due to constraints on the circuit depth by the inherent noise present in the hardware.

While JW calculations face a considerable handicap, compared to $\mathrm{P} 2 \mathrm{QR}$, in terms of qubit and total gate count, JW mapping generally has favorable gate count for the time evolution of the electronic structure Hamiltonian as the number of orbitals increases. As such, our five-qubit JW results serve as an important benchmark on the path to simulating larger systems.

We also employ the use of two reference states to extract the full spectra containing three singlets and a triplet state in this active space. This requires a total of 24 circuits (two reference states each require measurements of three matrix elements, each of which requires the real and imaginary parts of the one-body and two-body parts of the Hamiltonian) per geometry and each circuit is measured with $8 \times 10^{3}$ shots. Measurement error mitigation is also employed, and calibrated using $8 \times 10^{3}$ shots.

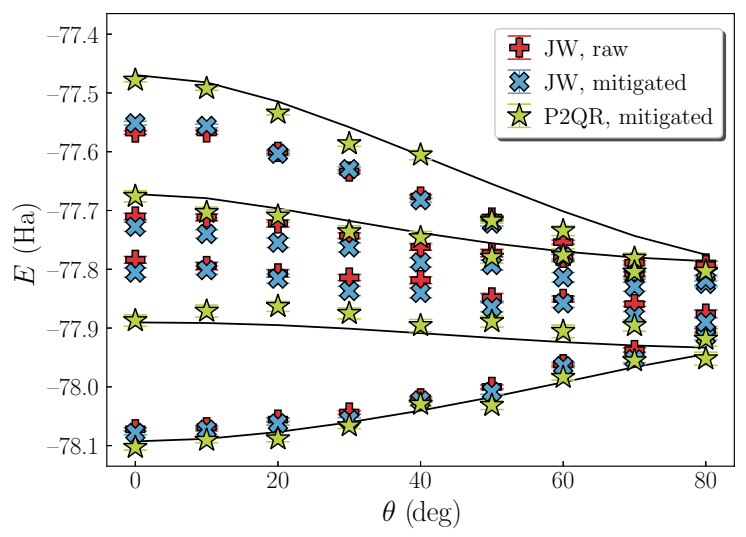

FIG. 15. Exact (black lines) and computed (colored points) energy spectra for twisted configurations of ethylene. Computations are performed on ibmq_mumbai, using JW (red plus symbols; blue crosses for raw, error-mitigated results) and P2QR (green stars) representations.
In Fig. 15, we explore the spectra of ethylene, as a function of torsion angle. Unsurprisingly, the P2QR results outperform the JW results, but they fail to accurately reflect the proper excited state energies when twisted more than $40^{\circ}$. Within the P2QR HOMO-LUMO active space, the number of particles in each spin species is automatically conserved, so our error mitigation strategies add no benefit.

The accuracy of the JW results follow a similar trend with the torsion angle, but induce larger systematic bias, as it requires deeper circuits. Implementing the postselection scheme within JW reduces the bias on the ground-state and lowest excited-state energies, but increases the bias on the other excited energies.

In Fig. 16, we report results for cis-stilbene, transstilbene, and $\mathrm{BChl} a$. Most of the $\mathrm{P} 2 \mathrm{QR}$ results are statistically compatible with exact energies, with slightly larger hardware errors on the highest energy in each of these experiments. As expected, the JW experiments yield larger systematic errors for the high-energy states. In the cis-stilbene plots it is clear that postselection provides a substantial correction to the first and second excited states. A similar effect is observed in the trans-stilbene case.

Conversely, in the BChl $a$ result, postselection mildly reduced the systematic error for the first excited state, but simultaneously shifted the second and third excited states further from their exact values. Given that there is a general trend for postselection to decrease the energies, it is likely that typical errors generate transitions to particle sectors with larger energies.

This still leaves open the source of error for the large bias in some of these data points. We suspect that noise on the ancilla qubit may dramatically affect the results, but leave a rigorous investigation of noise sources to future work.

\section{SUMMARY AND OUTLOOK}

In this work, we have considered a number of related techniques that can be stacked to substantially 

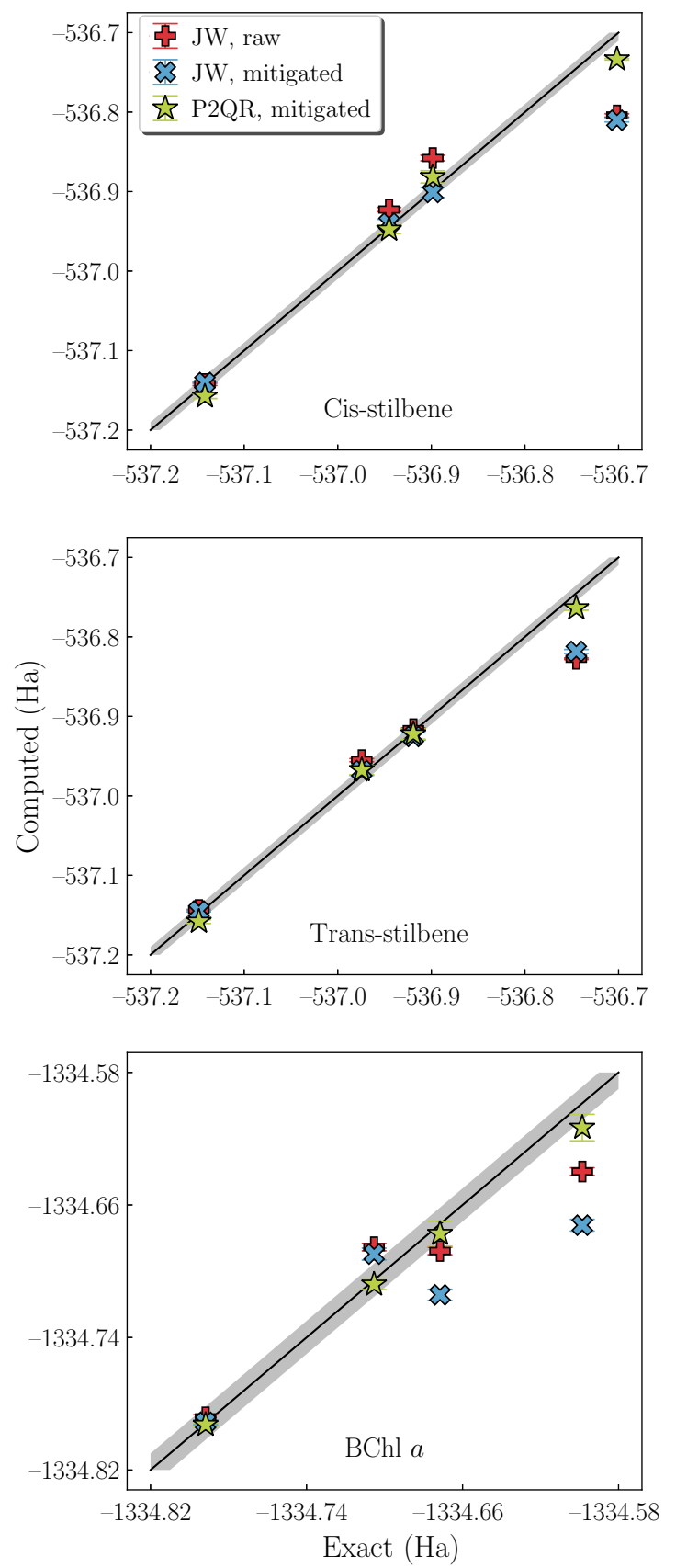

FIG. 16. Exact and computed energies for cis-stilbene, transstilbene, and BChl $a$ using the Jordan-Wigner representation (red, blue crosses for raw and corrected results) and parity representation with two-qubit reduction (green stars). The gray band has a width of $10 \mathrm{mHa}$. Computations are carried out on ibmq_mumbai and ibmq_guadalupe.

lower the quantum resources required to perform accurate computations of low-lying spectra of electronic Hamiltonians. The most straightforward step is the merger of the QFD approach with the low-rank DF representation of the electronic Hamiltonian, which provides considerable reductions of both the circuit size needed for
QFD time propagation and the number of measurements needed for Hamiltonian expectation value calculation. This merger of QFD and DF is further accelerated by intrinsic reductions in the required DF rank expansion afforded by moving from an X-DF representation to a C-DF representation. It is important to note that the choice of QFD in the present work was made to provide a practical demonstration of the C-DF representation, but it would be straightforward to also use C-DF with other methods for ground- or excited-state properties such as VQE [37], quantum subspace expansion VQE [9], MC VQE [38], subspace search VQE [39], non-orthogonal VQE [6], multi-reference selected quantum Krylov [4], VQPE [5], or QPE. Within these methods, reduced circuit depths will be obtained for Hamiltonian simulation steps and reduced required measurement costs will be obtained for Hamiltonian expectation values. For an example of how such speedups might be achieved, the interested reader is referred to the recent work by Burg et al. [40], which merged X-DF with qubitized QPE and achieved significant reduction in circuit depth. Here one could simply substitute the C-DF factors for the X-DF factors and achieve an additional speedup.

One interesting point that was approached but not fully solved within this work involves the positioning of double factorization between density fitting and tensor hypercontraction. Density fitting [41-49] and the closely related Cholesky decomposition approach for ERIs [5054] reduces the rank- 4 ERI tensor to a product of two rank-3 tensors $(p q \mid r s) \approx \sum_{A} L_{p q}^{A} L_{r s}^{A}$, where the auxiliary index size $n_{A}$ is found to scale linearly in $n_{p}$. Tensor hypercontraction $[28,55,56]$ reduces the ERI tensor to a product of five rank-2 tensors, with a structure $(p q \mid r s) \approx$ $\sum_{k l} X_{p k} X_{q k} Z_{k l} X_{r l} X_{s l}$ that is very similar to double factorization, but without the requirement that the leaf tensors $X_{p k}$ are orthogonal or square (i.e., $n_{k}$ may be different from, and usually larger than $n_{p}$, though is found to scale linearly in $n_{p}$ ). The nonorthogonality of tensor hypercontraction appears to be somewhat problematic for quantum algorithms, e.g., as evidenced by the need for linear combination of unitaries and qubitization approaches in a recent approach for the adoption of tensor hypercontraction into quantum algorithms by the Google team [57]. At the very least, the nonorthogonal operators of standard THC require additional conceptual and physical overhead to represent within quantum circuits, mandating that we seek an alternative unitary variant of THC. Explicit double factorization reduces the ERI tensor to an $n_{\mathrm{DF}}$-depth sum over unitary tensor hypercontractions, each indexed by $t$, i.e., $(p q \mid r s) \approx \sum_{t k l} U_{p k}^{t} U_{q k}^{t} Z_{k l}^{t} U_{r l}^{t} U_{s l}^{t}$ with the size of $n_{\mathrm{DF}}$ scaling linearly in $n_{p}$. The unitary nature of the leaf tensors $U_{p k}^{t}$ in double factorization makes the approach immediately amenable to implementation within quantum algorithms. However, explicit double factorization retains the rank-3 information content and cost of 
density fitting, rather than the rank- 2 information content and cost of tensor hypercontraction. It seems incongruous to us that simply constraining the tensor hypercontraction factorization to use unitary factors to facilitate deployment within quantum algorithms should cause a rise in the information content from rank 2 to rank 3 . This motivated our development of the compressed double factorization approach in this work as a pragmatic attempt to reduce the information content in the double factorization approach. Substantial numerical gains were demonstrated, but it is not clear if the resulting method achieves the constant $n_{\mathrm{DF}}$ depth required to obtain rank-2 information content. More work must be done to pursue an analog to the analytical exact tensor hypercontraction result [56] (which rigorously demonstrated the rank-2 information content of tensor hypercontraction) in closed basis sets, and to extend compressed double factorization to a more reliable and practical method for nonclosed basis sets.

We also investigated methods for circuit reduction and error mitigation to improve performance on noisy quantum hardware. The circuit reduction is performed by reorganizing our Hamiltonian so that the two-body DF terms only contain quadratic Pauli- $Z$ terms $\left(Z_{k, \sigma} Z_{l, \tau}\right)$ while simultaneously defining a new one-body term. When performing controlled time evolution, this process results in a saving of $\left(2 \times N \times n_{\mathrm{DF}}\right)$ CNOT gates, where $N$ is the number of qubits and $n_{\mathrm{DF}}$ is the number of double-factorized terms in the Hamiltonian.

In our measurement scheme we rotate to a diagonal basis of each factor of the double factorized C-DF Hamiltonian as shown in Fig. 9. This enables us to postselect only the results with the proper number of particles in each spin species. An extra layer of error mitigation can be employed by echoing with $\exp \left(-i \eta_{k, \sigma} \hat{N}_{\sigma}\right)$, as shown in Fig. 8. The combined benefit of both these mitigation strategies is demonstrated with the qasm noise simulation of the ethylene in Fig. 14. As the depth of these circuits increase, and the hardware noise is constrained to a modest level, the echo self-averaging effect can enable substantial error reduction within each individual instance. At shorter depths, it is important to average over random instances to smooth out the results. There are many other error mitigation strategies that can be implemented for these calculations. The benefit of the postselection and echo-sequencing schemes we present is that they naturally fit into the structure of these circuits with minimal resource overhead.

The methods presented in this work were also implemented on IBM's quantum devises with calculations of energy spectra for twisted ethylene, cis- and trans-stilbene, and $\mathrm{BChl} a$. As expected, calculation using $\mathrm{P} 2 \mathrm{QR}$ outperformed results that used JW mapping. The JW results were still able to modestly reproduce the proper energy spectra and should be seen as a benchmark for moving to larger systems.
The postselection scheme produced a modest improvement in the JW results and we still need to experiment with the echo sequences. It is clear that the noise-simulated backend does not faithfully emulate the true hardware noise in our experiments, which is to be expected as the simulated noise models are only meant to capture simple noise channels such as depolarization, amplitude damping, and bit flipping. This is readily seen when comparing the ethylene curves in Figs. 14 and 15. Given that the results are highly sensitive to noise on the ancilla qubit it will be important to investigate these noise sources further and determine how well they can mitigated.

Molecular structures and electronic Hamiltonian matrix elements are available from the authors upon reasonable request.

\section{ACKNOWLEDGMENTS}

The QC Ware effort in this work was supported by the U.S. Department of Energy, Office of Science, Basic Energy Sciences, Chemical Sciences, Geosciences and Biosciences Division. We thank an anonymous reviewer for a suggestion to more thoroughly explore the error characteristics of X-DF versus C-DF in the context of deviations in chemically important observables such as total adiabatic state energies.

R.M.P. owns stock and options in QC Ware Corp.

\section{APPENDIX A \\ 1. Example circuits}

In this section, we describe the detailed structure of the QFD circuits sketched in Figs. 8 and 9, focusing on a system of electrons in $M=2$ spatial orbitals, and using the Jordan-Wigner representation. In Fig. 17, we show the quantum circuit corresponding to a step of time evolution under the Hamiltonian, using a C-DF approximation of the ERI tensor with $n_{\mathrm{DF}}=1$ layers.

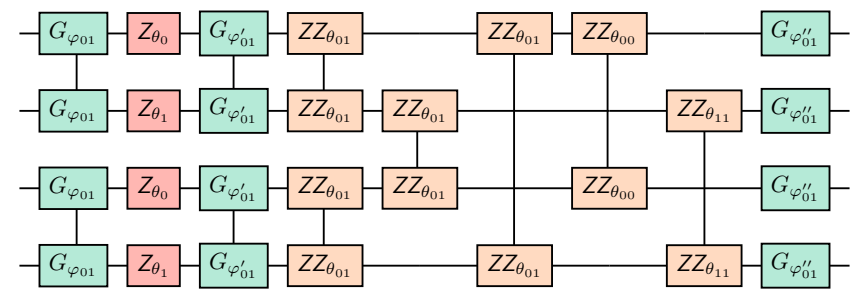

FIG. 17. Quantum circuit implementing a single step of time evolution under a Hamiltonian with $n_{\mathrm{DF}}=1$ layers acting on $M=2$ spatial orbitals in a Jordan-Wigner representation. Green, red, and orange blocks denote Givens rotations implementing basis changes, single-qubit $Z$ rotations, and two-qubit $Z$ rotations, respectively. 


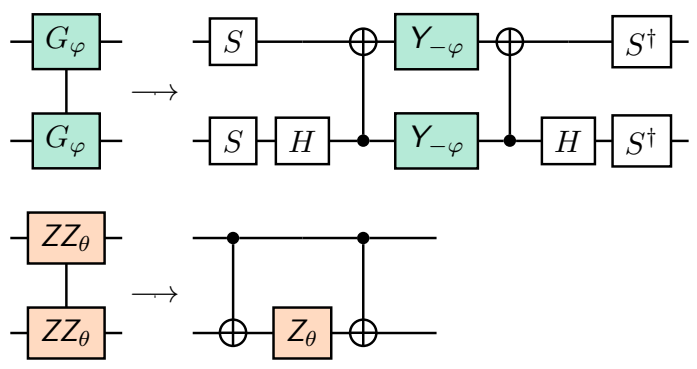

FIG. 18. Implementation of a Givens rotation (top) and of a two-qubit $Z$ rotation (bottom) with CNOT gates and single-qubit rotations.

The matrices $\hat{G}_{\mathrm{HF} \leftarrow t}$ and $\hat{G}_{t \leftarrow t^{\prime}}$, connecting eigenbases of the Fock and C-DF operators, are represented as products of Givens transformations with standard linear algebra techniques $[14,16]$. Givens transformations correspond to operators of the form

$$
\hat{G}_{r-1, r}\left(\varphi_{r-1, r}\right)=\prod_{\sigma} e^{-\varphi_{r-1, r}\left(\hat{a}_{r-1 \sigma}^{\dagger} \hat{a}_{r \sigma}-\hat{a}_{r \sigma}^{\dagger} \hat{a}_{r-1 \sigma}\right)},
$$

which, in a Jordan-Wigner representation, are represented by two quantum circuits (one for spin- $\alpha$ and one for spin$\beta$ particles), each acting on two qubits and comprising two CNOT gates, as shown in the upper portion of Fig. 18.

The representation of the Givens transformation as second-quantization operators, Eq. (A1), is also the starting point for deriving the corresponding quantum circuit under other representations of fermionic degrees of freedom with qubits (e.g., parity, Bravyi-Kitaev [58]), as well as in presence of qubit reduction techniques.

The one-body part of the Hamiltonian, on the other hand, is represented by the diagonal operator

$$
\hat{V}_{1 b}=e^{-i \Delta t \sum_{k \sigma} f_{k k^{\prime}}^{\prime} \hat{z}_{k \sigma}}=\prod_{k \sigma} e^{-i \Delta t f_{k}^{\prime} \hat{z}_{k \sigma}}
$$

Since, under the Jordan-Wigner representation, $\hat{z}_{k \uparrow} \rightarrow Z_{k}$ and $\hat{z}_{k \downarrow} \rightarrow Z_{k+M}$, the operation $\hat{V}_{1 b}$ is implemented by a product of $2 M$ single-qubit $\mathbf{Z}$ rotations with angles $\theta_{k}=$ $-2 \Delta t f_{k k}^{\prime}$,

$$
\hat{V}_{1 b} \rightarrow \prod_{k \sigma} \mathrm{Z}_{\theta_{k}, k \sigma}
$$

Such single-qubit operations are shown as red blocks in Fig. 17. For the same reason, the terms describing the twobody part of the Hamiltonian,

$$
\hat{V}_{2 b, t}=\prod_{k l, \sigma \tau}^{*} e^{-i \Delta t Z_{k l}^{t} \hat{z}_{k \sigma} \hat{z}_{l \tau} / 2}
$$

are implemented, in a Jordan-Wigner representation, by a product of $\mathcal{O}\left(M^{2}\right)$ two-qubit $Z Z$ rotations with angles $\theta_{k l}=$
$\Delta t Z_{k l}^{t}$

$$
\hat{V}_{2 b, t} \rightarrow \prod_{k l, \sigma \tau}^{*} \mathbf{Z Z}_{\theta_{k l}, k \sigma, l \tau}
$$

Such two-qubit transformations, shown as orange blocks in Fig. 17, are exponentials of the operator $Z \otimes Z$, and can thus be compiled into a product of two CNOT transformations and a single-qubit $Z$ rotation, as shown in the lower portion of Fig. 18.

It is useful to remark that the controlled version of $\hat{V}_{2 b, t}$ only requires to control the single-qubit $Z$ rotation, and not the two CNOT operations. Furthermore, a network of SWAP gates can be used to ensure that all $\mathbf{Z Z}$ and controlled$\mathrm{ZZ}$ rotations act on adjacent qubits (assuming linear chip topology) [16].

\section{DF energy convergence}

For reference, we study the convergence of the X-DF and C-DF methods in terms of correlation energy and singlet-triplet gaps for each of our reference molecules with an active space of $M=6$. In Fig. 19, we plot the differences in correlation energy and the singlet-triplet gap are with respect to $\operatorname{FCI}\left(n_{\mathrm{DF}}=\infty\right)$ in the $M=6$ active
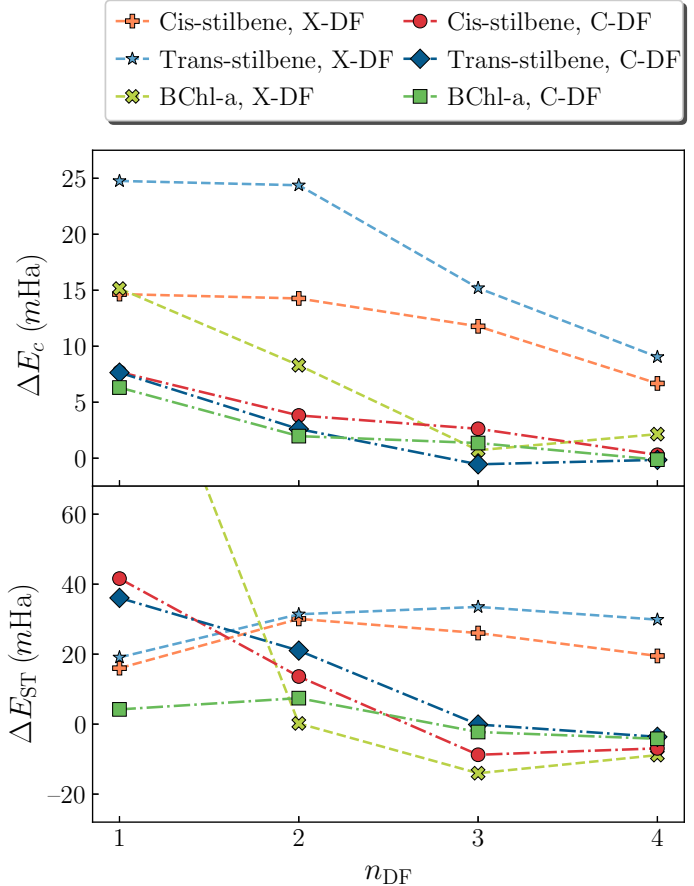

FIG. 19. Correlation energies (top) and singlet-triplet (ST) gaps (bottom) as a function of the number of layers for the X-DF and C-DF electron repulsion integrals (light, dark colors). Correlation energies and gaps are computed for the species studied in this work, with active spaces of $M=6$ orbitals, and values from the exact electron repulsion integral are used as reference. 
TABLE I. Deviations between exact and computed energies for ethylene. Energy deviations are computed as in the right portion of Fig. 14, averaged over torsion angle, and measured in milliHartrees.

\begin{tabular}{lcccc}
\hline \hline & $\Delta E_{\text {raw }}$ & $\Delta E_{\mathrm{ps}}$ & $\Delta E_{\text {echo }}$ & $\Delta E_{\mathrm{ps}+\text { echo }}$ \\
\hline$S_{0}$ & $24(11)$ & $4(7)$ & $3(4)$ & $1(2)$ \\
$S_{2}$ & $133(14)$ & $12(10)$ & $4(5)$ & $3(4)$ \\
$T_{1}$ & $14(19)$ & $9(12)$ & $11(6)$ & $2(3)$ \\
$S_{1}$ & $13(16)$ & $9(11)$ & $7(5)$ & $1(3)$ \\
\hline \hline
\end{tabular}

space of each molecule. When it comes to the correlation energies, the C-DF method yields more accurate estimates than X-DF for all values of $n_{\mathrm{DF}}$. Conversely, for the singlet-triplet gap, the X-DF only produces more accurate results when $n_{\mathrm{DF}}>2$.

\section{APPENDIX B: EFFECT OF POSTSELECTION AND ECHO SEQUENCING}

In Table I, we list the deviations between exact and computed energies for ethylene. Energies are computed as in Fig. 14, and averaged over the torsion angle for illustration purposes. As seen, use of postselection reduces the magnitude of the deviations in the case of $S_{2}$. Use of echo sampling, on the other hand, primarily reduces statistical uncertainties on energy deviations.

[1] J. Kempe, A. Kitaev, and O. Regev, The complexity of the local Hamiltonian Problem, SIAM J. Comput 35, 1070 (2006).

[2] I. M. Georgescu, S. Ashhab, and F. Nori, Quantum simulation, Rev. Mod. Phys. 86, 153 (2014).

[3] R. M. Parrish and P. L. McMahon, arXiv:1909.08925 (2019).

[4] N. H. Stair, R. Huang, and F. A. Evangelista, A multireference quantum krylov algorithm for strongly correlated Electrons, J. Chem. Theory Comput 16, 2236 (2020).

[5] K. Klymko, C. Mejuto-Zaera, S. J. Cotton, F. Wudarski, M. Urbanek, D. Hait, M. Head-Gordon, K. B. Whaley, J. Moussa, and N. Wiebe et al., arXiv:2103.08563 (2021).

[6] W. J. Huggins, J. Lee, U. Baek, B. O'Gorman, and K. B. Whaley, A non-orthogonal variational quantum eigensolver, New J. Phys 22, 073009 (2020).

[7] D. Neuhauser, Bound state eigenfunctions from wave packets: Time $\rightarrow$ energy resolution, J. Chem. Phys. 93, 2611 (1990).

[8] D. Neuhauser, Circumventing the heisenberg principle: A rigorous demonstration of filter-diagonalization on a liCN model, J. Chem. Phys. 100, 5076 (1994).

[9] J. R. McClean, M. E. Kimchi-Schwartz, J. Carter, and W. A. De Jong, Hybrid quantum-classical hierarchy for mitigation of decoherence and determination of excited states, Phys. Rev. A 95, 042308 (2017).
[10] M. Motta, C. Sun, A. T. Tan, M. J. O’Rourke, E. Ye, A. J. Minnich, F. G. Brandao, and G. K.-L. Chan, Determining eigenstates and thermal states on a quantum computer using quantum imaginary time evolution, Nat. Phys 16, 205 (2020).

[11] P. J. Ollitrault, A. Kandala, C.-F. Chen, P. K. Barkoutsos, A. Mezzacapo, M. Pistoia, S. Sheldon, S. Woerner, J. M. Gambetta, and I. Tavernelli, Quantum equation of motion for computing molecular excitation energies on a noisy quantum processor, Phys. Rev. Res. 2, 043140 (2020).

[12] D. Poulin, M. B. Hastings, D. Wecker, N. Wiebe, A. C. Doberty, and M. Troyer, The Trotter step size required for accurate quantum simulation of quantum chemistry, Quant. Info. Comput 15, 361 (2015).

[13] B. Peng and K. Kowalski, Highly efficient and scalable compound decomposition of two-electron integral tensor and its application in coupled cluster Calculations, J. Chem. Theory Comput 13, 4179 (2017).

[14] M. Motta, E. Ye, J. R. McClean, Z. Li, A. J. Minnich, R. Babbush, and G. K. Chan, arXiv:1808.02625 (2018).

[15] M. Motta, J. Shee, S. Zhang, and G. K.-L. Chan, Efficient ab initio auxiliary-field quantum monte carlo calculations in Gaussian bases via low-rank tensor Decomposition, J. Chem. Theory Comput 15, 3510 (2019).

[16] I. D. Kivlichan, J. McClean, N. Wiebe, C. Gidney, A. Aspuru-Guzik, G. K.-L. Chan, and R. Babbush, Quantum simulation of electronic structure with linear depth and Connectivity, Phys. Rev. Lett 120, 110501 (2018).

[17] D. W. Berry, C. Gidney, M. Motta, J. R. McClean, and R. Babbush, Qubitization of arbitrary-basis quantum chemistry leveraging sparsity and low-rank factorization, Quantum 3, 208 (2019).

[18] Y. Matsuzawa and Y. Kurashige, Jastrow-type decomposition in quantum chemistry for low-depth quantum Circuits, J. Chem. Theory Comput 16, 944 (2020).

[19] W. J. Huggins, J. R. McClean, N. C. Rubin, Z. Jiang, N. Wiebe, K. B. Whaley, and R. Babbush, Efficient and noise resilient measurements for quantum chemistry on near-term quantum computers, Npj Quantum Inf. 7, 1 (2021).

[20] T. Helgaker, P. Jorgensen, and J. Olsen, Molecular Electronic-Structure Theory (John Wiley \& Sons, New York, 2014).

[21] P. Gokhale, O. Angiuli, Y. Ding, K. Gui, T. Tomesh, M. Suchara, M. Martonosi, and F. T. Chong, $\mathrm{O}\left(\mathrm{N}^{3}\right)$ measurement cost for variational quantum eigensolver on molecular Hamiltonians, IEEE Trans. Quantum Eng. 1, 1 (2020).

[22] V. Verteletskyi, T.-C. Yen, and A. F. Izmaylov, Measurement optimization in the variational quantum eigensolver using a minimum clique cover, J. Chem. Phys. 152, 124114 (2020).

[23] Note that this notation of double factorization $\rightarrow$ DF presents an unfortunate elision with the popular and related density fitting representation, which is often denoted as DF in the literature. To avoid any issues, we explicitly write out "density fitting" for the few times it is encountered in this manuscript.

[24] M. Reck, A. Zeilinger, H. J. Bernstein, and P. Bertani, Experimental realization of any discrete unitary operator, Phys. Rev. Lett 73, 58 (1994). 
[25] D. Wecker, M. B. Hastings, N. Wiebe, B. K. Clark, C. Nayak, and M. Troyer, Solving strongly correlated electron models on a quantum computer, Phys. Rev. A 92, 062318 (2015).

[26] F. Arute et al., (Google AI Quantum), Hartree-fock on a superconducting qubit quantum computer, Science 369, 1084 (2020).

[27] R. M. Wilcox, Exponential operators and parameter differentiation in quantum Physics, J. Math. Phys 8, 962 (1967).

[28] R. M. Parrish, E. G. Hohenstein, T. J. Martínez, and C. D. Sherrill, Tensor hypercontraction. II. Least-squares renormalization, J. Chem. Phys. 137, 224106 (2012).

[29] M. Head-Gordon and J. A. Pople, Optimization of wave function and geometry in the finite basis hartree-fock method, J. Phys. Chem. 92, 3063 (1988).

[30] D. Aharonov, V. Jones, and Z. Landau, in Proc STOC (ACM, 2006), p. 427.

[31] M. C. Tran, Y. Su, D. Carney, and J. M. Taylor, Phys. Rev. X Quantum 2, 010323 (2021).

[32] X. Bonet-Monroig, R. Sagastizabal, M. Singh, and T. O'Brien, Low-cost error mitigation by symmetry verification, Phys. Rev. A 98, 062339 (2018).

[33] S. McArdle, X. Yuan, and S. Benjamin, Error-mitigated digital quantum Simulation, Phys. Rev. Lett 122, 180501 (2019).

[34] G. Aleksandrowicz, T. Alexander, P. Barkoutsos, L. Bello, Y. Ben-Haim, D. Bucher, F. Cabrera-Hernández, J. Carballo-Franquis, A. Chen, and C. Chen et al., Zenodo (2019).

[35] A. W. Cross, L. S. Bishop, S. Sheldon, P. D. Nation, and J. M. Gambetta, Validating quantum computers using randomized model circuits, Phys. Rev. A 100, 032328 (2019).

[36] ibmq_guadalupe v1.3.1, ibmq_montreal v1.3.1, and ibmq_ mumbai v1.3.3, IBM Quantum Team, Retrieved from https://quantum-computing.ibm.com (2020).

[37] A. Peruzzo, J. McClean, P. Shadbolt, M.-H. Yung, X.-Q. Zhou, P. J. Love, A. Aspuru-Guzik, and J. L. O'brien, A variational eigenvalue solver on a photonic quantum processor, Nat. Commun. 5, 1 (2014), arXiv:1304. 3061.

[38] R. M. Parrish, E. G. Hohenstein, P. L. McMahon, and T. J. Martínez, Quantum Computation of Electronic Transitions Using a Variational Quantum Eigensolver, Phys. Rev. Lett. 122, 230401 (2019).

[39] K. M. Nakanishi, K. Mitarai, and K. Fujii, Subspace-search variational quantum eigensolver for excited states, Phys. Rev. Res. 1, 033062 (2019).

[40] V. von Burg, G. H. Low, T. Häner, D. S. Steiger, M. Reiher, M. Roetteler, and M. Troyer, arXiv:2007.14460 (2020).

[41] J. L. Whitten, Coulombic potential energy integrals and approximations, J. Chem. Phys. 58, 4496 (1973).

[42] B. I. Dunlap, J. W. D. Connolly, and J. R. Sabin, On the applicability of LCAO-X $\alpha$ methods to molecules containing transition metal atoms: The nickel atom and nickel hydride, Int. J. Quantum Chem. Symp. 12, 81 (1977).
[43] B. I. Dunlap, J. W. D. Connolly, and J. R. Sabin, On some approximations in applications of $\mathrm{X} \alpha$ theory, J. Chem. Phys. 71, 3396 (1979).

[44] M. Feyereisen, G. Fitzgerald, and A. Komornicki, Use of approximate integrals in ab initio theory. An application in MP2 energy calculations, Chem. Phys. Lett. 208, 359 (1993).

[45] A. Komornicki and G. Fitzgerald, Molecular gradients and hessians implemented in density functional theory, J. Chem. Phys. 98, 1398 (1993).

[46] O. Vahtras, J. Almlöf, and M. W. Feyereisen, Integral approximations for LCAO-SCF calculations, Chem. Phys. Lett. 213, 514 (1993).

[47] A. P. Rendell and T. J. Lee, Coupled-cluster theory employing approximate integrals: An approach to avoid the input/output and storage bottlenecks, J. Chem. Phys. 101, 400 (1994).

[48] R. A. Kendall and H. A. Fruchtl, The impact of the resolution of the identity approximate integral method on modern ab initio algorithm development, Theor. Chem. Acc. 97, 158 (1997).

[49] F. Weigend, A fully direct RI-HF algorithm: Implementation, optimised auxiliary basis sets, demonstration of accuracy and efficiency, Phys. Chem. Chem. Phys. 4, 4285 (2002).

[50] N. H. F. Beebe and J. Linderberg, Simplifications in the generation and transformation of two-electron integrals in molecular calculations, Int. J. Quantum Chem. 12, 683 (1977).

[51] I. Roeggen and E. Wisloff-Nilssen, On the beebe-linderberg two-electron integral approximation, Chem. Phys. Lett. 132, 154 (1986).

[52] H. Koch, A. S. de Meras, and T. B. Pedersen, Reduced scaling in electronic structure calculations using cholesky decompositions, J. Chem. Phys. 118, 9481 (2003).

[53] F. Aquilante, T. B. Pedersen, and R. Lindh, Low-cost evaluation of the exchange fock matrix from cholesky and density fitting representations of the electron repulsion integrals, J. Chem. Phys. 126, 194106 (2007).

[54] F. Aquilante, L. Gagliardi, T. B. Pedersen, and R. Lindh, Atomic cholesky decompositions: A route to unbiased auxiliary basis sets for density fitting approximation with tunable accuracy and efficiency, J. Chem. Phys. 130, 154107 (2009).

[55] E. G. Hohenstein, R. M. Parrish, and T. J. Martínez, Tensor hypercontraction density fitting. I. Quartic scaling secondand third-order Møller-plesset perturbation theory, J. Chem. Phys. 137, 044103 (2012).

[56] R. M. Parrish, E. G. Hohenstein, N. F. Schunck, C. D. Sherrill, and T. J. Martínez, Exact Tensor Hypercontraction: a universal technique for the resolution of matrix elements of local finite-range N-body potentials in many-body quantum problems, Phys. Rev. Lett 111, 132505 (2013).

[57] J. Lee, D. Berry, C. Gidney, W. J. Huggins, J. R. McClean, N. Wiebe, and R. Babbush, arXiv:2011.03494 (2020).

[58] S. B. Bravyi and A. Y. Kitaev, Fermionic quantum Computation, Ann. Phys 298, 210 (2002). 